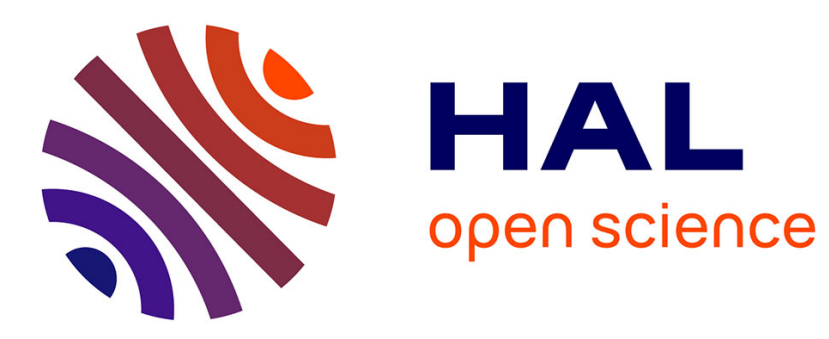

\title{
Feedforwarding under sampling
}

Mattia Mattioni, Salvatore Monaco, Dorothée Normand-Cyrot

\section{To cite this version:}

Mattia Mattioni, Salvatore Monaco, Dorothée Normand-Cyrot. Feedforwarding under sampling. IEEE Transactions on Automatic Control, In press, 64 (11), 10.1109/TAC.2019.2902666 . hal01923758

\section{HAL Id: hal-01923758 \\ https://hal.science/hal-01923758}

Submitted on 15 Nov 2018

HAL is a multi-disciplinary open access archive for the deposit and dissemination of scientific research documents, whether they are published or not. The documents may come from teaching and research institutions in France or abroad, or from public or private research centers.
L'archive ouverte pluridisciplinaire HAL, est destinée au dépôt et à la diffusion de documents scientifiques de niveau recherche, publiés ou non, émanant des établissements d'enseignement et de recherche français ou étrangers, des laboratoires publics ou privés. 


\title{
Feedforwarding under sampling
}

\author{
Mattia Mattioni ${ }^{1}$, Salvatore $_{\text {Monaco }}{ }^{1}$ and Dorothée Normand-Cyrot ${ }^{2}$
}

\begin{abstract}
The paper deals with stabilization of feedforward multiple cascade dynamics under sampling. It is shown that $u$-average passivity concepts and Lyapunov methods can be profitably exploited to provide a systematic sampled-data design procedure. The proposed methodology recalls the continuous-time feedforwarding steps and can be applied under the same assumptions as those set over the continuous-time cascade dynamics. The final sampled feedback is carried out through a three steps procedure that involves iterative passivation and stabilization in the $u$-average sense. Constructive aspects are developed to compute approximate solutions which are indeed implemented in practice. An example is worked out upon the paper with comparative simulations with respect to usual sampled-and-hold implementations.
\end{abstract}

\section{Index Terms}

Nonlinear systems, Sampled data control, Algebraic/geometric methods, Stability of NL systems

\section{INTRODUCTION}

$\mathbf{S}$ INCE the very first work on backstepping [1], nonlinear constructive control has been providing a prolific field of investigation for stabilizing nonlinear systems admitting suitable triangular structures ( [2]-[4]). The consequent feedback control laws are rather easy to compute and yield robustness in closed loop as usual when relying upon Lyapunov-based methodologies.

Forwarding based-design has been introduced as the dual of backstepping for dynamics in the so-called feedforward form ( [5]-[9]). The design exploits the cascade structure for defining a Lyapunov function via the construction of a suitable cross term dominating all the terms with non-definite sign. Then, passivity arguments are used to achieve stabilization in closed loop. When specified over strict-feedforward structures, such a procedure recovers the idea of recursively introducing a state component which integrates the other ones ( [5], [10]). Intriguing connections with stabilization over invariant sets can also be set in the framework of Immersion and Invariance [11]. This class of cascade systems embeds a lot of cases from different scenarios so allowing a constructive design even in complex practical situations also involving observer design (e.g., [12]-[17]).

All of this concerns continuous-time dynamics while a very few works have been addressing the problem in discrete time. In this case, things get complicated because of the loss of a geometrical framework sustaining the evolutions and, even more important, the nonlinearities with respect to the control variables of the mappings and equations defining the solutions. These difficulties motivate important contributions ( [18]-[21]) mostly devoted to strict-feedforward dynamics. More recently, a solution for general feedforward structures has been proposed in discrete time in [22], [23].

In between, a challenging perspective is provided by the sampled-data scenario ( [24]-[28]); namely, when the control is piecewise constant and measures are available at the sampling instants only. In this context, stabilization of cascade systems at large cannot proceed along the same lines as in continuous time. As a matter of fact, in most cases (e.g., [29], [30] for strict-feedback dynamics), the cascade structure is destroyed by sampling so hardly compromising the iterative nature of the design. A particular case is provided by upper triangular (feedforward) dynamics that indeed preserve the cascade structure under sampling. Though, applying the feedforwarding procedure presented in [22], [23] for purely discrete-time systems might be quite conservative as it does not take advantage of the properties yielded by the original continuous-time plant. In addition, further assumptions than the continuous-time ones are needed. In the digital literature, several works have been proposed when restricting to classes of feedforward dynamics or when considering sample-and-hold solutions. In [31], the authors consider feedforward systems that are minimum phase with respect to a given output and basically work out the design in continuous time; implementation is then performed through usual emulation by proving its efficacy under sampling for small values of the sampling period. Similar results are in [32], [33] where the authors also provide an explicit bound to the sampling period preserving stability in closed loop.

At the best of the authors' knowledge, a unifying framework for forwarding design under sampling exploiting the hybrid nature of the overall system is still missing. Thus, the present paper aims at bridging this gap. Roughly speaking, the underlying idea of the approach we propose goes beyond the idea of looking for control solutions (parametrized by the sampling period) aimed at reproducing the same performances as in continuous time. As a matter of fact, the design relies upon the definition of a feedback solution that is still parametrized by the sampling period but designed over new $\delta$-dependent performances,

Mattia Mattioni thanks the Université Franco-Italienne/Università Italo-Francese (UFI/UIF) for supporting his mobility from France to Italy within his PhD program.

${ }^{1}$ Dipartimento di Ingegneria Informatica, Automatica e Gestionale A. Ruberti (La Sapienza University of Rome); Via Ariosto 25, 00185 Rome, Italy \{mattia.mattioni, salvatore.monaco\}euniromal.it.

${ }^{2}$ Laboratoire de Signaux et Systèmes (L2S, CNRS); 3, Rue Joliot Curie, 91192, Gif-sur-Yvette, France cyrot@l2s. centralesupelec.fr 
criteria and properties inferred from the continuous-time ones. The case of $u$-average passivity represents a paradigm of this new family of strategies.

Specifically, we provide a systematic procedure for stabilizing the feedforward interconnection of continuous-time dynamics via sampled-data state feedback. We prove that a redefinition of the constructive mappings and Lyapunov functions is needed to solve the problem under sampling with no extra assumption than the continuous-time ones. The sampled-data design we propose is constructive and proceeds in three steps over a suitable two-block dynamics: first, a preliminary sampled-data feedback asymptotically stabilizing the lower component is described; then, a Lyapunov function is constructed over the closed-loop double cascade dynamics which is also shown to be stable; finally, asymptotic stabilization of the whole twoblock system is achieved via u-average passivity around a nominal feedback solution. These three steps are repeated over a new double cascade system issued by successively interconnecting one upper dynamics. To this end, we extend the notion of $u$-average passivity as introduced in [34] for purely discrete-time systems to deal with incremental-like properties that are essential for the iterative nature of the design.

The preliminary work in [35] treats the two block cascade case when assuming, in continuous time, the first element of the dynamics to possess a globally asymptotically stable and locally exponentially stable equilibrium in free evolution. Here, this assumption is weakened so enabling the extension of the result to multiple cascade interconnections. Without loss of generality, we discuss the result for single-input systems while the generalization to the multi-input case follows along the same lines.

The reminder of the paper follows. First, basics on sampled-data systems are given in Section III Then, the continuoustime feedforwarding procedure is recalled in Section IV] where the problem is settled as well. In Section V] the sampled-data forwarding design is detailed on an elementary two blocks cascade interconnection. Its extension to the general case is provided in Section VI through an algorithmic procedure. Constructive aspects are developed in Section VII to work out an executive way of computing approximate solutions for practical implementation issues. An example is carried out through the dissertation with comparative simulations. Section VIII concludes the paper.

\section{NOTATIONS AND DEFINITIONS}

$M_{\mathbb{U}}$ denotes the space of measurable and locally bounded functions $u: \mathbb{R}^{+} \rightarrow \mathbb{U}$, with $\mathbb{U} \subseteq \mathbb{R}$ and $M_{\mathbb{U}}^{I}$ the space of measurable and locally bounded functions $u: I \rightarrow \mathbb{U}$, with $I \subset \mathbb{R}$. The set

$$
\mathscr{U}_{\delta}=\left\{u \in M_{\mathbb{U}} \text { s.t. } u(t)=u_{k}, \forall t \in[k \delta,(k+1) \delta[\text { and } k \geq 0\}\right.
$$

denotes the set of piecewise constant functions over time intervals of length $\delta$, a finite time interval $\delta \in] 0, T[$.

Maps are assumed smooth and vector fields complete. Given a vector field $f, \mathrm{~L}_{f}$ denotes the associated Lie derivative operator, $\mathrm{L}_{f}=\sum_{i=1}^{n} f_{i}(\cdot) \nabla_{x_{i}}$ with $\nabla_{x_{i}}=\frac{\partial}{\partial x_{i}}$ and $\nabla=\left(\nabla_{x_{1}} \ldots \nabla_{x_{n}}\right)$. Given two vector fields $f$ and $\mathrm{g}$, we define the Lie bracket $a d_{f} g=$ $(\nabla g) f-(\nabla f) g$ and iteratively $a d_{f}^{i} g=a d_{f}\left(a d_{f}^{i-1} g\right)$ with $a d_{f}^{0} g=g$. Given a mapping $H: \mathbb{R}^{n} \rightarrow \mathbb{R}$ and a constant $\bar{x} \in \mathbb{R}^{n}$, we denote $\nabla H(\bar{x})=\left.\nabla H(x)\right|_{x=\bar{x}}$.

$e^{\mathrm{L}_{f}}$ is the usual exponential Lie series operator, $e^{\mathrm{L}_{f}}=\mathrm{I}+\sum_{i \geq 1} \frac{\mathrm{L}_{f}^{i}}{i !}$ so that for any function $h: \mathbb{R}^{n} \rightarrow \mathbb{R}^{p}$, one gets $h\left(\left.e^{\mathrm{L}_{f}} \mathrm{Id}\right|_{x}\right)=$ $e^{\mathrm{L}_{f}} h(x)=h(x)+\sum_{i \geq 1} \frac{1}{i !} \mathrm{L}_{f}^{i} h(x)$ where Id and I denote respectively the identity function and identity operator.

A function $\gamma(\cdot):[0, \infty) \rightarrow[0, \infty)$ is said to be of class $\mathscr{K}$ if it is zero in zero and strictly increasing; if, moreover, it is unbounded, it is said of class $\mathscr{K}_{\infty}$. A function $\omega(z, \xi)$ is said to satisfy a linear growth property with respect to the first variable if there exist functions $\gamma_{1}(\cdot), \gamma_{2}(\cdot) \in \mathscr{K}$ differentiable at $\xi=0$, such that $\|\omega(z, \xi)\| \leq \gamma_{1}(\|\xi\|)\|z\|+\gamma_{2}(\|\xi\|)$. A function $R(x, \delta)=O\left(\delta^{p}\right)$ is said of order $\delta^{p}, p \geq 1$ if whenever it is defined it can be written as $R(x, \delta)=\delta^{p-1} \tilde{R}(x, \delta)$ and there exist a function $\theta \in \mathscr{K}_{\infty}$ and $\delta^{*}>0$ s. t. $\forall \delta \leq \delta^{*},|\tilde{R}(x, \delta)| \leq \theta(\delta)$.

\section{BASICS ON SAMPLED-DATA SYSTEMS}

Consider an input-affine dynamics

$$
\dot{x}(t)=f(x(t))+g(x(t)) u(t), \quad x \in \mathbb{R}^{n}, u \in M_{\mathbb{U}}
$$

and assume the control piecewise constant over intervals of length $\delta$ (i.e., $u \in \mathscr{U}_{\delta}$ ) and measures of the state available only at the sampling instants $t=k \delta$. In such a context, (1) rewrites as the interval dynamics

$$
\dot{x}(t)=f(x(t))+g(x(t)) u_{k}, \quad t \in[k \delta,(k+1) \delta[.
$$




\section{A. Sampled-data equivalent models}

The sampled-data equivalent model to $(1)$ is obtained through integration of 2 over $\delta$ with initial condition $x_{k}=x(k \delta)$. The associated difference equations get the form of a map $F^{\delta}\left(\cdot, u_{k}\right): \mathbb{R}^{n} \times \mathbb{R} \rightarrow \mathbb{R}^{n}$

$$
x_{k+1}=F^{\delta}\left(x_{k}, u_{k}\right)=\left.e^{\delta\left(\mathrm{L}_{f}+u_{k} \mathrm{~L}_{g}\right)} x\right|_{x_{k}}=x_{k}+\left.\sum_{i \geq 1} \frac{\delta^{i}}{i !} \mathrm{L}_{f+g u_{k}}^{i} x\right|_{x_{k}} .
$$

As well known, $F^{\delta}(\cdot, u)$ is nonlinear in the control variable $u$ and parameterized by the sampling period $\delta$ ( [36], [37]). As closed-form sampled-data models cannot be exactly computed in general, one makes reference to approximations by truncating the power series 3 at any finite order in $\delta^{p}$ with $p \in \mathbb{N}$. As an example, when neglecting the terms in $O\left(\delta^{2}\right)$, one recovers the Euler approximation of $3 x_{k+1}=x_{k}+\delta\left(f\left(x_{k}\right)+g\left(x_{k}\right) u_{k}\right)$ commonly considered in the literature as it indeed preserves most of the continuous-time properties [38], [39].

The $\left(F_{0}, G\right)$ representation has been proposed in [40] as an alternative to 3 (namely, of the mapping $F^{\delta}(\cdot, u)$ ). Denoting by $x^{+}(u)$ any curve in $\mathbb{R}^{n}$ parameterized by $u \in \mathbb{R}$, one defines the differential/difference form of 3 as

$$
\begin{aligned}
x^{+} & =F_{0}^{\delta}(x), \quad x^{+}=x^{+}(0) \\
\frac{\mathrm{d} x^{+}(u)}{\mathrm{d} u} & =G^{\delta}\left(x^{+}(u), u\right)
\end{aligned}
$$

with

$$
F_{0}^{\delta}(x)=e^{\delta \mathrm{L}_{f}} x ; \quad G^{\delta}(x, u)=\int_{0}^{\delta} e^{-s a d_{f+u g}} g(x) \mathrm{d} s .
$$

Specifically, the map $F_{0}^{\delta}(x)=F^{\delta}(x, 0)$ describes the free evolution of the dynamics when $u=0$ while the $u$-dependent vector field $G^{\delta}(x, u)$ over $\mathbb{R}^{n}$ models the variation of the map $F^{\delta}(\cdot, u)$ with respect to the control and around $F^{\delta}(\cdot, 0)$.

In the sampled-data context, both representations are perfectly equivalent. More in detail, given any pair $\left(x_{k}, u_{k}\right)$ for $k \geq 0$, one recovers the usual difference equation (3) by integrating (4b] over $u \in\left[0, u_{k}[\right.$ with initial condition (4a); i.e., one gets the following equality (see [40] for complete proofs and computational aspects)

$$
F^{\delta}\left(x_{k}, u_{k}\right)=F_{0}^{\delta}\left(x_{k}\right)+\int_{0}^{u_{k}} G^{\delta}\left(x^{+}(v), v\right) \mathrm{d} v
$$

and, thus, $x_{k+1}=x^{+}\left(u_{k}\right)=F^{\delta}\left(x_{k}, u_{k}\right)$.

The $\left(F_{0}, G\right)$ representation is useful to carry out analysis and control design over sampled dynamics in a differential geometric framework as illustrated in the sequel in terms of passivity or passivation.

It is important to emphasize that, when the same initial condition $x(0)=x_{0}$ is assumed, the trajectories of (3) (and, equivalently, (4)) coincide for any $k \geq 0$ with the ones of (2) at any sampling instant $t=k \delta$. Thus, properties of the sampled-data system (3) (and, equivalently, (4)) are equivalent to the properties of the continuous-time dynamics (2) at any $t=k \delta, k \geq 0$. In this sense, we recall the following definition about stabilization at the sampling instants.

Definition 3.1 (S-GAS and S-LES): The equilibrium of the sampled dynamics 22 is sampled-data GAS (S-GAS) (resp. sampled-data LES, S-LES) under a suitable piecewise constant $u_{k}=u\left(x_{k}\right)$ if it is GAS (resp. LES) for the closed-loop discretetime equivalent dynamics $x_{k+1}=F^{\delta}\left(x_{k}, u\left(x_{k}\right)\right)$.

\section{B. Sampled-data average-passivity}

The notion of $u$-average passivity has been introduced in [34] when referring to a discrete-time system. Let $\Sigma_{\delta}$ be a generic sampled-data system described by the dynamics 4 with output map $H(\cdot, u): \mathbb{R}^{n} \rightarrow \mathbb{R}$ (possibly depending on $u$ ). $u$-average passivity specifies as follows.

Definition 3.2 (u-average passivity): $\Sigma_{\delta}$ is $u$-average passive if there exists a $C^{1}$ function $S(\cdot): \mathbb{R}^{n} \rightarrow \mathbb{R}_{\geq 0}$ (the storage function) such that for any pair $\left(x_{k}, u_{k}\right)(k \geq 0)$, one verifies the inequality

$$
S\left(x^{+}\left(u_{k}\right)\right)-S\left(x_{k}\right) \leq H^{a v}\left(x_{k}, u_{k}\right) u_{k}
$$

where

$$
H^{a v}(x, u)=\frac{1}{u} \int_{0}^{u} H\left(x^{+}(v), v\right) d v
$$

defines the u-average output map associated to $H(x, u)$.

Remark 3.1: The dissipativity inequality (5) underlines that whenever $\Sigma_{\delta}$ is $u$-average passive, it is passive (in the usual sense) with respect to the average output $H^{a v}(x, u)$. 
According to the $\left(F_{0}, G\right)$ representation, the average passivity inequality 5 rewrites in its integral form as follows

$$
S\left(F_{0}^{\delta}\left(x_{k}\right)\right)-S\left(x_{k}\right)+\int_{0}^{u_{k}} \mathrm{~L}_{G^{\delta}(\cdot, v)} S\left(x^{+}(v)\right) \mathrm{d} v \leq H^{a v}\left(x_{k}, u_{k}\right) u_{k}
$$

because for any pair $\left(x_{k}, u_{k}\right)$, the first order difference $\Delta_{k} S(x)=S\left(x_{k+1}\right)-S\left(x_{k}\right)=S\left(x^{+}\left(u_{k}\right)\right)-S\left(x_{k}\right)$ rewrites as

$$
\Delta_{k} S=S\left(F_{0}^{\delta}\left(x_{k}\right)\right)-S\left(x_{k}\right)+\int_{0}^{u_{k}} \mathrm{~L}_{G^{\delta}(\cdot, v)} S\left(x^{+}(v)\right) \mathrm{d} v
$$

Exploiting (6), the extended concept of $u$-average passivity from some nominal control value $\bar{u}$ is here introduced.

Definition 3.3 (u-average passivity from/around $\bar{u}$ ): $\Sigma_{\delta}$ is u-average passive from $\bar{u} \in \mathbb{R}$ if there exists a $C^{1}$ function $S(\cdot)$ : $\mathbb{R}^{n} \rightarrow \mathbb{R}_{\geq 0}$ (the storage function) such that, for any pair $\left(x_{k}, u_{k}\right)$ and $k \geq 0$, one verifies the inequality

$$
\Delta_{k} S=S\left(x^{+}(\bar{u})\right)-S\left(x_{k}\right)+\int_{\bar{u}}^{u_{k}} \mathrm{~L}_{G^{\delta}(\cdot, v)} S\left(x^{+}(v)\right) \mathrm{d} v \leq \int_{\bar{u}}^{u_{k}} H\left(x^{+}(v), v\right) \mathrm{d} v .
$$

Remark 3.2: The notion of $u$-average passivity from $\bar{u}$ is strictly reminiscent of the concept of incremental passivity [41]. It defines incremental-like passivity of the overall system with respect to trajectories that are parametrized by different inputs $u$ rather than time. Current work is addressing this aspect.

Remark 3.3: $u$-average passivity from $\bar{u}$ can be understood as $u$-average passivity of the dynamics under the preliminary control $\bar{u}$; namely, one has

$$
\int_{\bar{u}}^{u_{k}} H\left(x^{+}(v), v\right) \mathrm{d} v=\int_{0}^{u_{k}-\bar{u}} H\left(x^{+}(\bar{u}+v), \bar{u}+v\right) \mathrm{d} v .
$$

Defining

$$
H_{\bar{u}}^{a v}\left(x_{k}, u_{k}\right)=\frac{1}{u_{k}-\bar{u}} \int_{\bar{u}}^{u_{k}} H\left(x^{+}(v), v\right) \mathrm{d} v
$$

the inequality (7) rewrites as $\Delta_{k} S(x) \leq\left(u_{k}-\bar{u}\right) H_{\bar{u}}^{a v}\left(x_{k}, u_{k}\right)$ so recovering, when $\bar{u}=0$, $u$-average passivity with the average output $H_{0}^{a v}(x, u)=H^{a v}(x, u)$.

\section{FEEDFOWARD SYSTEMS AND FORWARDING: RECALLS}

Let the continuous-time feedforward dynamics

$$
\begin{array}{ll}
\dot{z}=f(z)+\varphi(z, \xi)+g(z, \xi) u, & z \in \mathbb{R}^{n_{z}} \\
\dot{\xi}=a(\xi)+b(\xi) u, & \xi \in \mathbb{R}^{n_{\xi}}, u \in \mathbb{R}
\end{array}
$$

possess an equilibrium at the origin and assume the standard feedforwarding assumptions [2].

Assumption 4.1: The functions $\varphi(z, \xi)$ and $g(z, \xi)$ satisfy the linear growth property with respect to the state $z$.

Assumption 4.2: $\dot{z}=f(z)$ is globally stable (GS), with radially unbounded and locally quadratic Lyapunov function $W(z)$ so that $\mathrm{L}_{f} W(z) \leq 0$ for all $z$. Moreover, there exist real constants $c$ and $M$ such that, for $\|z\|>M$,

$$
\|\nabla W(z)\|\|z\| \leq c W(z)
$$

Assumption 4.3: $\dot{\xi}=a(\xi)$ is globally stable (GS), with radially unbounded and locally quadratic Lyapunov function $U(\xi)$ such that $\mathrm{L}_{a} U(\xi) \leq 0$ for any $\xi$.

The next Theorem is recalled from [2].

Theorem 4.1: Let the cascade dynamics (8) verify Assumptions 4.1 to 4.3 and the sub-dynamics $8 \mathrm{~b}$ with output $y_{0}=\mathrm{L}_{b} U(\xi)$ be Zero State Detectable (ZSD) 1 Let the pair $(\nabla a(0), b(0))$ be stabilizable. Then:

1) the feedback $u_{0}=-\mathrm{L}_{b} U(\xi)$ makes the equilibrium of $8 \mathrm{~b}$ globally asymptotically stable (GAS) and locally asymptotically stable (LES);

2) there exists a continuous cross term

$$
\Psi(z, \xi)=\int_{0}^{\infty} \mathrm{L}_{\varphi(\cdot, \xi(s))-g(\cdot, \xi(s)) \mathrm{L}_{b} U(\xi(s))} W(z(s)) \mathrm{d} s
$$

\footnotetext{
${ }^{1}$ Given a continuous-time dynamics $\dot{x}=f(x)+g(x) u$ with output map $h(\cdot): \mathbb{R}^{n} \rightarrow \mathbb{R}^{n}$, let $Z \subset \mathbb{R}^{n}$ the largest positively invariant set when $u=0$, contained in $\left\{x \in \mathbb{R}^{n} \mid h(x)=0\right\}$. Then, the system is said ZSD if $x=0$ is asymptotically stable conditionally to $Z$.
} 
evaluated along the solutions of the globally stable dynamics

$$
\begin{aligned}
& \dot{z}=f(z)+\varphi(z, \xi)-g(z, \xi) \mathrm{L}_{b} U(\xi) \\
& \dot{\xi}=a(\xi)-b(\xi) \mathrm{L}_{b} U(\xi)
\end{aligned}
$$

making $V(z, \xi)=U(\xi)+\Psi(z, \xi)+W(z)$ a radially unbounded Lyapunov function for [10;;

3) the dynamics 8 with output $y=\mathrm{L}_{\bar{g}} V(z, \xi)^{2}$ is passive with storage function $V(z, \xi)$;

4) the control law $u=-\mathrm{L}_{\bar{g}} V(z, \xi)$ achieves GAS of the equilibrium. If the Jacobian linearization of [8] is stabilizable, such a feedback ensures LES of the equilibrium.

Under Assumptions 4.1 to 4.3 the damping feedback $u_{0}=-\mathrm{L}_{b} U(\xi)$ makes the equilibrium of $(8)$ globally stable. Accordingly, one deduces the existence of a cross term of the form (9) satisfying the partial derivative equation

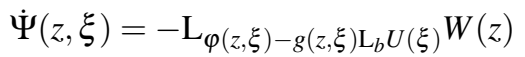

along the trajectories of 10 so that the Lyapunov function $V(z, \xi)=U(\xi)+\Psi(z, \xi)+W(z)$ is non-increasing along the closed-loop dynamics; i.e.

$$
\left.\dot{V}\right|_{u=-\mathrm{L}_{b} U(\xi)}=\mathrm{L}_{f} W(z)+\left.\dot{U}(\xi)\right|_{u=-\mathrm{L}_{b} U(\xi)} \leq-\frac{1}{2}\left\|\mathrm{~L}_{b} U(\xi)\right\|^{2}
$$

\section{A. Feedforward dynamics under sampling}

Detailing the sampled-data equivalent models in Section III-A to $[8$ and setting $x=\operatorname{col}(z, \xi), \bar{f}(x)=\operatorname{col}(f(z)+\varphi(z, \xi), a(\xi))$, $\bar{g}(x)=\operatorname{col}(g(z, \xi), b(\xi))$, one gets the following result.

Lemma 4.1: The sampled-data equivalent model to $(8)$ preserves the feedforward structure; i.e. in the form of a map

$$
\begin{aligned}
& z_{k+1}=f^{\delta}\left(z_{k}\right)+\varphi^{\delta}\left(z_{k}, \xi_{k}\right)+g^{\delta}\left(z_{k}, \xi_{k}, u_{k}\right) \\
& \xi_{k+1}=a^{\delta}\left(\xi_{k}, u_{k}\right)
\end{aligned}
$$

with

$$
\begin{aligned}
a^{\delta}(\xi, u) & =e^{\delta\left(\mathrm{L}_{a}+u \mathrm{~L}_{b}\right)} \xi ; \quad f^{\delta}(z)=e^{\delta \mathrm{L}_{f}} z \\
\varphi^{\delta}(z, \xi) & =\delta \varphi(z, \xi)+\sum_{i \geq 1} \frac{\delta^{i+1}}{(i+1) !} \varphi_{i}(z, \xi) \\
g^{\delta}(z, \xi, u) & =\delta g(z, \xi) u+\sum_{i \geq 1} \frac{\delta^{i+1}}{(i+1) !} g_{i}(z, \xi, u) \\
\varphi_{i}(z, \xi) & =\mathrm{L}_{\bar{f}}^{i}\left(\mathrm{~L}_{f}+\mathrm{L}_{\varphi}\right) z-\mathrm{L}_{f}^{i+1} z \\
g_{i}(z, \xi, u) & =u \mathrm{~L}_{\bar{g}}\left(\mathrm{~L}_{f}^{i-1} z+\varphi_{i-1}(z, \xi)+g_{i-1}(z, \xi, u)\right)
\end{aligned}
$$

Lemma 4.2: The $\left(F_{0}, G\right)$ form equivalent to (12) exhibits a feedforward structure as described below

$$
\begin{aligned}
z^{+} & =F_{0}^{\delta}(z, \xi) \\
\frac{\mathrm{d} z^{+}(u)}{\mathrm{d} u} & =G^{\delta}\left(z^{+}(u), \xi^{+}(u), u\right) \\
\xi^{+} & =a_{0}^{\delta}(\xi) \\
\frac{\mathrm{d} \xi^{+}(u)}{\mathrm{d} u} & =B^{\delta}\left(\xi^{+}(u), u\right)
\end{aligned}
$$

where

$$
\begin{aligned}
& F_{0}^{\delta}(z, \xi)=f^{\delta}(z)+\varphi^{\delta}(z, \xi) ; \quad a_{0}^{\delta}(\xi)=a^{\delta}(\xi, 0) \\
& G^{\delta}(z, \xi, u)=\int_{0}^{\delta} e^{-\operatorname{sad}_{\bar{f}+u \bar{g}} g(z, \xi) \mathrm{d} s} \\
& B^{\delta}(\xi, u)=\int_{0}^{\delta} e^{-\operatorname{sad}_{a+u b} b(\xi) \mathrm{d} s}
\end{aligned}
$$

When necessary, one compactly rewrites 13 as

$$
x^{+}=\bar{F}_{0}^{\delta}(x) ; \quad \frac{\mathrm{d} x^{+}(u)}{\mathrm{d} u}=\bar{G}^{\delta}\left(x^{+}(u), u\right)
$$

with $\bar{F}_{0}^{\delta}(\cdot)=\operatorname{col}\left(F_{0}^{\delta}(\cdot), a_{0}^{\delta}(\cdot)\right)$ and $\bar{G}^{\delta}(\cdot)=\operatorname{col}\left(G^{\delta}(\cdot), B^{\delta}(\cdot)\right)$. 


\section{B. Problem statement}

We address the problem of designing a sampled-data feedback making the closed-loop equilibrium of (8) S-GAS. In doing so, we exploit the preservation of the feedforward structure of (8) under sampling and make extensive use of Lyapunov and $u$-average passivity arguments to deduce the control law. Moreover, only the continuous-time assumptions of Theorem 4.1 are shown to be sufficient to fulfill the goal.

\section{SAMPLED-DATA STABILIZATION OF FEEDFORWARD DYNAMICS}

Given (8) verifying the assumptions set in Theorem 4.1, the following three items will be proven over its sampled-data equivalent model $[12)$ :

1) there exists a feedback $u=u_{0}^{\delta}(\xi)$ (or simply $u_{0}^{\delta}$ when no confusion arises) ensuring GAS and LES of the equilibrium of the $\xi$-dynamics $12 \mathrm{~b}$ (Theorem 5.1 ;

2) a new and explicitly $\delta$-dependent Lyapunov function $V^{\delta}(\cdot): \mathbb{R}^{n_{z}} \times \mathbb{R}^{n_{\xi}} \rightarrow \mathbb{R}_{>0}$ can be constructed for the augmented dynamics (12) (Proposition 5.1);

3) there exists an output mapping $Y^{\delta}(z, \xi, u)$ so that 12 is $u$-average passive from $u_{0}^{\delta}$ and ZSD with storage function $V^{\delta}(z, \xi)$; accordingly, one can construct a sampled-data feedback ensuring GAS and LES of the equilibrium of the complete cascade (12) (Theorem 5.2).

These three items will be repeated to deal with multiple cascade in Section VI so to get S-GAS of the overall (8).

Remark 5.1: As sketched in the introduction, all of the criteria and mappings involved in the passivation-based design and Lyapunov analysis are, in general, different from the continuous-time ones although no further hypotheses are needed to ensure their existence.

\section{A. Stabilization of the $\xi$-subsystem}

Given (8) with sampled-data equivalent model $[12$, , let us first stabilize the $\xi$-dynamics $12 \mathrm{~b}$ through passivity-based design in the $u$-average sense [34].

Theorem 5.1: Let $8 \mathrm{~b}$ ) satisfy Assumption 4.3 and be ZSD with respect to the output $y_{0}=\mathrm{L}_{b} U(\xi)$ and assume the linear pair $(\nabla a(0), b(0))$ stabilizable. Then, the sampled-data system $[12 \mathrm{~b}$ (equivalently, $13 \mathrm{c}-(13 \mathrm{~d})$ ) with output

$$
Y_{0}^{\delta}(\xi, u)=\frac{1}{\delta} \mathrm{L}_{B^{\delta}(\cdot, u)} U(\xi)
$$

is $u$-average passive. Moreover, the control $u=u_{0}^{\delta}(\xi)$ solution to the equality

$$
u=-Y_{0}^{\delta, \text { av }}(\xi, u)
$$

makes the closed-loop equilibrium of (8b) S-GAS and S-LES.

Proof: From Assumption 4.3 one verifies that, for any $\xi_{k}=\xi(k \delta), k \geq 0$ and $u=0$

$$
\begin{aligned}
U\left(\xi_{k}^{+}(0)\right)-U\left(\xi_{k}\right) & =U\left(a_{0}^{\delta}\left(\xi_{k}\right)\right)-U\left(\xi_{k}\right) \\
& =\int_{k \delta}^{(k+1) \delta} \mathrm{L}_{a} U(\xi(s)) \mathrm{d} s \leq 0
\end{aligned}
$$

over the free evolution $\dot{\xi}=a(\xi)$ and at any sampling instant $t=k \delta$. Substituting this inequality into the increment

$$
\Delta_{k} U(\xi)=U\left(\xi_{k}^{+}(0)\right)-U\left(\xi_{k}\right)+\int_{0}^{u_{k}} \mathrm{~L}_{B^{\delta}(\cdot, v)} U\left(\xi^{+}(v)\right) \mathrm{d} v
$$

one gets $u$-average passivity with respect to [14]; i.e.

$$
\Delta_{k} U(\xi) \leq u_{k}\left(\frac{1}{u_{k}} \int_{0}^{u_{k}} \mathrm{~L}_{B^{\delta}(\cdot, v)} U\left(\xi^{+}(v)\right) \mathrm{d} v\right)=\delta u_{k} Y_{0}^{\delta, \text { av }}\left(\xi, u_{k}\right) .
$$

GAS of the equilibrium under the feedback 15 follows provided $12 \mathrm{~b}$ with output mapping $Y_{0}^{\delta}(\xi, 0)$ is ZSD. To this end, it is easily verified that the largest positively invariant set contained in $\left\{x \in \mathbb{R}^{n} \mid \mathrm{L}_{B^{\delta}(\cdot, 0)} U\left(\xi^{+}(0)\right)=0\right\}$ coincides with the largest positively invariant set contained in $\left\{x \in \mathbb{R}^{n} \mid \mathrm{L}_{b} U(\xi)=0\right\}$. Furthermore, when $u=0$ the sampled-data and continuous-time trajectories coincide at any sampling instants. Thus, stability of $\xi_{k+1}=a_{0}^{\delta}(\xi)$, conditionally to $Z$, follows. Finally LES of $(12 \mathrm{~b})$ (equivalently, (13c)-(13d) ) is implied by stabilizability in first approximation of $(8 \mathrm{~b})$. $\triangleleft$

Remark 5.2: The output (14) making the sampled-data system $12 \mathrm{~b}$-average passive is different from the continuous-time one since it is explicitly dependent on the control and smoothly parametrized by the sampling period. More specifically, it rewrites as a series expansion in powers of $\delta$ of the form

$$
Y_{0}^{\delta}(\xi, u)=\mathrm{L}_{b} U(\xi)-\frac{\delta}{2} \mathrm{~L}_{a d_{a} b} U(\xi)+\frac{\delta^{2}}{3 !} \mathrm{L}_{a+u b} \mathrm{~L}_{a d_{a} b} U(\xi)+O\left(\delta^{3}\right)
$$

so getting $Y_{0}^{\delta}(\xi, u) \rightarrow \mathrm{L}_{b} U(\xi)$ as $\delta \rightarrow 0$ (i.e., the continuous-time passivating output). 


\section{B. A Lyapunov function for the augmented cascade}

Let us now consider the closed-loop dynamics $(8)$ under piecewise constant feedback $u_{k}=u_{0}^{\delta}\left(\xi_{k}\right)$ defined in $[15]$ which is governed, for $t \in[k \delta,(k+1) \delta[$, by the differential equations

$$
\begin{aligned}
& \dot{z}(t)=f(z)+\varphi(z, \xi)+g(z, \xi) u_{0}^{\delta}\left(\xi_{k}\right) \\
& \dot{\xi}(t)=a(\xi)+b(\xi) u_{0}^{\delta}\left(\xi_{k}\right) .
\end{aligned}
$$

In the sequel, we investigate on the existence of a Lyapunov function for 16 of the form

$$
V^{\delta}(z, \xi)=U(\xi)+\Psi^{\delta}(z, \xi)+W(z)
$$

where the cross term $\Psi^{\delta}(z, \xi)$ is defined so to ensure, at any sampling instant, $\Delta_{k} V^{\delta}(z, \xi) \leq 0$ along the trajectories of 16 .

The cross term $\Psi^{\delta}(\cdot)$ is, in general, different from the continuous-time one $\Psi(\cdot)$ in $(9$; this is motivated by the fact that the closed-loop trajectories of $(16)$ and $(10)$ differ at the sampling instants. However, the existence of $\Psi^{\delta}(z, \xi)$ can be proven under the same Assumptions 4.1 4.2 and 4.3 as in continuous time. As detailed in Section VII-B, its construction can be worked out through its series expansion in powers of $\delta$ of the form

$$
\Psi^{\delta}(z, \xi)=\Psi_{0}(z, \xi)+\sum_{i \geq 1} \frac{\delta^{i}}{(i+1) !} \Psi_{i}(z, \xi)
$$

around the continuous-time solution $\Psi_{0}(z, \xi)=\Psi(z, \xi)$.

Before stating the result, let us note that, when defining $V^{\delta}(\cdot)$ as in 17 , the inequality below is easily verified along the trajectories of 16

$$
\begin{aligned}
\Delta_{k} V^{\delta}(z, \xi) & =\Delta_{k} \Psi^{\delta}(z, \xi)+\Delta_{k} W(z)+\Delta_{k} U(\xi) \\
& \leq \Delta_{k} \Psi^{\delta}(z, \xi)+\int_{k \delta}^{(k+1) \delta} \mathrm{L}_{\varphi+u_{0}^{\delta}\left(\xi_{k}\right) g} W(z(s), \xi(s)) \mathrm{d} s
\end{aligned}
$$

because, by Assumption 4.2. $\mathrm{L}_{f} W(z) \leq 0$ and $\Delta_{k} U(\xi) \leq 0$ by construction of $u_{0}^{\delta}\left(\xi_{k}\right)$ in Theorem 5.1 It follows that for guaranteeing that $V^{\delta}(\cdot)$ is non increasing, the cross term $\Psi^{\delta}(\cdot)$ should satisfy the equality

$$
\Delta_{k} \Psi^{\delta}(z, \xi)=-\int_{k \delta}^{(k+1) \delta} \mathrm{L}_{\varphi+u_{0}^{\delta}\left(\xi_{k}\right) g} W(z(s), \xi(s)) \mathrm{d} s
$$

along the trajectories of 16 .

Proposition 5.1: Let (8) verify Assumptions 4.1 to 4.3 Then, the solutions of $(16)$ are bounded at any $k \geq 0$ and $(19)$ admits a solution $\Psi^{\delta}(\cdot): \mathbb{R}^{n_{z}} \times \mathbb{R}^{n_{\xi}} \rightarrow \mathbb{R}$ of the form

$$
\Psi^{\delta}(z, \xi)=\sum_{\ell=0}^{\infty} \int_{\ell \delta}^{(\ell+1) \delta} \mathrm{L}_{\varphi+u_{0}^{\delta}\left(\xi_{\ell}\right) g} W(z(s), \xi(s)) \mathrm{d} s
$$

that is continuous. Furthermore, $V^{\delta}(z, \xi): \mathbb{R}^{n_{z}} \times \mathbb{R}^{n_{\xi}} \rightarrow \mathbb{R}$ defined as in 17 is a positive definite and radially unbounded Lyapunov function for 16 at any sampling instant $t=k \delta, k \geq 0$ and, equivalently, for 12 under $u_{k}=u_{0}^{\delta}\left(\xi_{k}\right)$ as in 15 .

Proof: The proof of the existence of the sampled-data cross term follows the lines of the continuous-time case [2]. It is achieved by showing that the $z$-dynamics remains bounded for $k \geq 0$ and that the terms in the sum in the right hand side of 20] are positive and bounded. As far as boundedness of $z$ is concerned, from Assumption 4.1 one computes

$$
\Delta_{k} W(z) \leq \int_{k \delta}^{(k+1) \delta}\|\nabla W(z(s))\|\left(\gamma_{0}(\|\xi(s)\|)+\gamma_{1}(\|\xi(s)\|)\|z(s)\|\right) \mathrm{d} s
$$

for some $\mathscr{K}$-functions $\gamma_{0}(\cdot)$ and $\gamma_{1}(\cdot)$. Moreover, because of LES of $16 \mathrm{a}$, there exists a $\mathscr{K}$-function $\gamma(\cdot)$ such that

$$
\Delta_{k} W(z) \leq \int_{k \delta}^{(k+1) \delta}\|\nabla W(z(s))\|(\gamma(\|\xi(s)\|)+\gamma(\|\xi(s)\|)\|z(s)\|) \mathrm{d} s .
$$

From now on, let us assume that $\|z(s)\|>1$ in the sampling interval (the result is straightforward if not). Accordingly, one gets

$$
\Delta_{k} W(z) \leq c \int_{k \delta}^{(k+1) \delta} \gamma(\|\xi(s)\|) W(z(s)) \mathrm{d} s .
$$

By construction of $u_{0}^{\delta}(\cdot)$ one has $\Delta_{k} U(\xi) \leq-\delta\left\|Y_{0}^{\delta \text {,av }}(\xi)\right\|^{2}$ ensuring GAS and LES of the closed-loop equilibrium of $12 \mathrm{~b}$; namely, when $\xi$ is sufficiently close to the origin

$$
\left\|\xi_{k+1}\right\| \leq e^{-\alpha \delta k} \gamma_{\xi}(\|\xi\|)
$$


for some $\mathscr{K}$-function $\gamma_{\xi}(\cdot)$, constant $\alpha>0$ and initial condition $\xi_{0}=\xi$. As a consequence, by rewriting [21] as

$$
\int_{k \delta}^{(k+1) \delta} \dot{W}(z(s)) \mathrm{d} s \leq c \int_{k \delta}^{(k+1) \delta} \gamma(\|\xi(s)\|) W(z(s)) \mathrm{d} s .
$$

and exploiting LES of the closed-loop equilibrium of $[12 \mathrm{~b}]$, one gets

$$
W\left(z_{k+1}\right) \leq e^{c \delta\left(e^{-\alpha \delta}-1\right) \gamma\left(\left\|\xi_{k}\right\|\right)} W\left(z_{k}\right)
$$

so proving $\Delta_{k} W(z) \leq 0$ and thus GS of the equilibrium of $[12$ in closed-loop.

Since $W(z)$ is radially unbounded, one has that $z$ and $\|\nabla W(z)\|$ stay bounded for $k \geq 0$. Thus, one deduces for a suitable $\lambda^{\delta} \in(0,1)$

$$
\int_{k \delta}^{(k+1) \delta} \mathrm{L}_{\varphi+u_{0}^{\delta} g} W(z(s), \xi(s)) \mathrm{d} s \leq \delta \gamma_{1}(\|(z, \xi)\|) \lambda^{k \delta}
$$

so concluding that $\int_{k \delta}^{(k+1) \delta} \mathrm{L}_{\varphi+u_{0}^{\delta}\left(\xi_{k}\right) g} W(z(s), \xi(s)) \mathrm{d} s$ is summable for $k \geq 0$ and that 20 exists and is bounded for all bounded $(z, \xi)$. The remaining part of the proof follows the lines of the discrete-time one as developed in [22], [23] in discrete time. $\triangleleft$

Remark 5.3: The equality (19) rewrites in integral-differential form as

$$
\int_{k \delta}^{(k+1) \delta} \mathrm{L}_{\bar{f}+u_{0}^{\delta}\left(\xi_{k}\right) \bar{g}} \Psi^{\delta}(z(s), \xi(s)) \mathrm{d} s=-\int_{k \delta}^{(k+1) \delta} \mathrm{L}_{\varphi+u_{0}^{\delta}\left(\xi_{k}\right) g} W(z(s), \xi(s)) \mathrm{d} s
$$

so extending to the sampled-data context the partial differential equation (11). In Section VII-B, it will be instrumental to express the integral-differential equation 19$]$ as an infinite number of partial differential equations.

Remark 5.4: The construction of the cross term might be carried out by considering the sampled-data equivalent model (12) under $u_{k}=u_{0}^{\delta}\left(\xi_{k}\right)$ as a purely discrete-time system [23]. Namely, one would look for a Lyapunov function $V_{d}(z, \xi)=$ $U(\xi)+W(z)+\Psi_{d}(z, \xi)$ where the new cross term should be chosen to satisfy the equality

$$
\left.\Delta_{k} \Psi_{d}(z, \xi)\right|_{u=u_{0}^{\delta}(\xi)}=-W\left(f^{\delta}(z)+\varphi^{\delta}(z, \xi)+g^{\delta}\left(z, \xi, u_{0}^{\delta}(\xi)\right)\right)+W\left(f^{\delta}(z)\right) .
$$

The above equality is in general different and more conservative than 19 and its solvability requires further assumptions than the continuous-time ones. As a matter of fact, (22) does not take into account the continuous-time nature of the plant and the properties of the original vector fields defining its dynamics. Specifically, the discrete-time approach tends to erase even the terms in the right-hand side of 22 whose sign is well-defined because of Assumption 4.2 as one gets

$$
\int_{k \delta}^{(k+1) \delta} \mathrm{L}_{f} W(z(s)) \mathrm{d} s=W\left(f^{\delta}(z)\right)-W(z)+\Theta^{\delta}(z, \xi) \leq 0
$$

where the term

$$
\Theta^{\delta}(z, \xi)=\int_{k \delta}^{(k+1) \delta} \int_{0}^{\xi} \nabla_{v}\left(e^{s \mathrm{~L}_{\bar{f}(\cdot, v)}} \mathrm{L}_{f(\cdot)} W(z)\right) \mathrm{d} v \mathrm{~d} s+\int_{k \delta}^{(k+1) \delta} \int_{0}^{u_{0}^{\delta}(\xi)} \nabla_{u}\left(e^{s \mathrm{~L}_{\bar{f}(, \xi \xi)}+u \mathrm{~L}_{\bar{g}(\cdot, \xi)}} \mathrm{L}_{f(\cdot)} W(z)\right) \mathrm{d} u \mathrm{~d} s
$$

is erased by 22, although the corresponding contribution in $\Delta_{k} V_{d}(z, \xi)$ is negative definite.

Remark 5.5: Equalities (19) and (22) come to coincide whenever the dynamics is in strict-feedforward form (i.e., when $f(z)=F z$ and the coupling vector fields do not depend on $z$ ). In that case, the construction of the cross term follows the lines of the discrete-time case by underlying some geometric properties yielded by the design. For the sake of space, it will be omitted (see [22], [23] for further details).

\section{Sampled-data passivity-based feedforwarding}

Once a Lyapunov function $V^{\delta}(z, \xi)$ is constructed over $\sqrt{16}$, one can verify that the sampled dynamics 12 (or, equivalently, 13) is $u$-average passive from $u_{0}^{\delta}(\xi)$ with respect to a suitably defined output mapping. For this purpose, when $u=u_{0}^{\delta}(\xi)$, one considers the inequality

$$
\Delta_{k} V^{\delta}(z, \xi) \leq U\left(\xi^{+}\left(u_{0}^{\delta}(\xi)\right)\right)-U(\xi)+\int_{u_{0}^{\delta}(\xi)}^{u} \mathrm{~L}_{\bar{G}^{\delta}(\cdot, v)} V^{\delta}\left(z^{+}(v), \xi^{+}(v)\right) \mathrm{d} v
$$

with

$$
U\left(\xi^{+}\left(u_{0}^{\delta}(\xi)\right)\right)-U(\xi) \leq-\delta\left\|Y_{0}^{\delta, \text { av }}(\xi)\right\|^{2}
$$

by construction of $u_{0}^{\delta}(\xi)$ and with

$$
\xi^{+}\left(u_{0}^{\delta}(\xi)\right)=a^{\delta}\left(\xi, u_{0}^{\delta}(\xi)\right)
$$


Accordingly, any controller $u$ making the second part of the right hand side of (23) negative achieves GAS of the equilibrium of the complete cascade $[12$. This is resumed in the main Theorem below.

Theorem 5.2: Let $(8)$ verify Assumptions 4.1 to 4.3 Let $8 \mathrm{~b}$ with output $y_{0}=\mathrm{L}_{b} U(\xi)$ be ZSD and stabilizable in first approximation. Then, the following holds:

1) the sampled-data equivalent dynamics (12) with output

$$
Y_{1}^{\delta}(z, \xi, u)=\frac{1}{\delta} \mathrm{L}_{\bar{G}}^{\delta(\cdot, u)} V^{\delta}(z, \xi)
$$

is $u$-average passive from $u_{0}^{\delta}(z, \xi)$ as in 15 with storage function $V^{\delta}(z, \xi)$ in $(17)$;

2) the feedback law $u=u_{1}^{\delta}(z, \xi)$ solution to the implicit equality

$$
u=-Y_{u_{0}^{\delta}(\xi)}^{\delta, \text { av }}(z, \xi, u)
$$

makes the closed-loop equilibrium of (8) S-GAS;

$3)$ if the dynamics $(8 \mathrm{~b})$ is stabilizable in first approximation, $u=u_{1}^{\delta}(z, \xi)$ makes the equilibrium of $(8) \mathrm{S}$-LES.

Proof: Rewriting (23) as

$$
\Delta_{k} V^{\delta} \leq-\delta\left\|Y_{0}^{\delta, \text { av }}\left(\xi, u_{0}^{\delta}(\xi)\right)\right\|^{2}+\delta u Y_{u_{0}^{\delta}(\xi)}^{\delta, \text { av }}(z, \xi, u)+\delta Y_{0}^{\delta, \text { av }}\left(\xi, u_{0}^{\delta}(\xi)\right) Y_{u_{0}^{\delta}(\xi)}^{\delta, \text { av }}(z, \xi, u)
$$

with by definition

$$
Y_{u_{0}^{\delta}(\xi)}^{\delta, \text { av }}(z, \xi, u)=\frac{1}{u-u_{0}^{\delta}(\xi)} \int_{u_{0}^{\delta}(\xi)}^{u} Y_{1}^{\delta}\left(z^{+}(v), \xi^{+}(v), v\right) \mathrm{d} v
$$

one deduces (through the Triangle inequality 3 ,

$$
\begin{aligned}
\Delta_{k} V^{\delta}(z, \xi) \leq & -\frac{\delta}{2}\left\|Y_{0}^{\delta, \text { av }}\left(\xi, u_{0}^{\delta}(\xi)\right)\right\|^{2}+\delta u Y_{u_{0}^{\delta}(\xi)}^{\delta, \text { av }}(z, \xi, u) \\
& +\frac{\delta}{2}\left\|Y_{u_{0}^{\delta}(\xi)}^{\delta, \text { av }}(z, \xi, u)\right\|^{2} .
\end{aligned}
$$

The feedback law 24 enables to conclude

$$
\Delta_{k} V^{\delta}(z, \xi) \leq-\frac{\delta}{2}\left\|Y_{0}^{\delta, \text { av }}\left(\xi, u_{0}^{\delta}\right)\right\|^{2}-\frac{\delta}{2}\left\|Y_{u_{0}^{\delta}(\xi)}^{\delta, \text { av }}\left(z, \xi, u_{1}^{\delta}\right)\right\|^{2} .
$$

This implies that the largest invariant set contained into $\left\{(z, \xi) \in \mathbb{R}^{n_{z}} \times \mathbb{R}^{n_{\xi}}:\left\|Y_{0}^{\delta, \text { av }}\left(\xi, u_{0}^{\delta}(\xi)\right)\right\|+\left\|Y_{u_{0}^{\delta}(\xi)}^{\delta, \text { av }}\left(z, \xi, u_{1}^{\delta}(z, \xi)\right)\right\| \equiv 0\right\}$ coincides with the largest invariant set contained into $\left\{\xi \in \mathbb{R}^{n_{\xi}}:\left\|Y_{0}^{\delta, \text { av }}\left(\xi, u_{0}^{\delta}(\xi)\right)\right\| \equiv 0\right\}$. From ZSD of $8 \mathrm{~b}$ with respect to $y_{0}=\mathrm{L}_{b} U(\xi)$, GAS of the closed-loop equilibrium of 12 under 24 follows. $\triangleleft$

Remark 5.6: From equality 24, , it can be verified that when $z \equiv 0$, one recovers $u_{1}^{\delta}(0, \xi)=u_{0}^{\delta}(\xi)$.

\section{THE CASE OF MULTIPLE CASCADE INTERCONNECTION}

The procedure here presented extends to multiple interconnected feedforward dynamics of the form

$$
\begin{aligned}
& \dot{z}^{i}=f^{i}\left(z^{i}\right)+\varphi^{i}\left(z^{i}, \ldots, z^{1}, \xi\right)+g^{i}\left(z^{i}, \ldots, z^{1}, \xi\right) u \\
& \dot{\xi}=a(\xi)+b(\xi) u
\end{aligned}
$$

with $z^{i} \in \mathbb{R}^{n_{z}}$ and $i=1, \ldots, N$. Accordingly, we suppose that Assumptions 4.1 to 4.3. with required extensions, hold on the vector fields defining the dynamics 25 .

Assuming that the linearization of (25) at the origin is stabilizable, then GAS and LES of the closed-loop equilibrium can be achieved trough an iterative procedure by proving passivity (in the $u$-average sense), at each step $i$, with respect the output $Y_{i}^{\delta}(\cdot)$ deduced from the corresponding Lyapunov function $V_{i}^{\delta}(\cdot)$. Given $[25$, let

$$
\begin{aligned}
z^{i+} & =f_{i}^{\delta}\left(z^{i}\right)+\varphi_{i}^{\delta}\left(z^{i}, \ldots, z^{1}, \xi\right), \\
\frac{\mathrm{d} z^{i+}(u)}{\mathrm{d} u} & =G_{i}^{\delta}\left(z^{i+}(u), \ldots, z^{1+}(u), \xi^{+}(u), u\right) \\
\xi^{+} & =a_{0}^{\delta}(\xi) \\
\frac{\mathrm{d} \xi^{+}(u)}{\mathrm{d} u} & =B^{\delta}\left(\xi^{+}(u), u\right)
\end{aligned}
$$

${ }^{3} p q \leq \frac{1}{2 \varepsilon} p^{2}+\frac{\varepsilon}{2} q^{2}$ for $\varepsilon>0$ 
be the $\left(F_{0}, G\right)$ representation of its sampled-data equivalent model. Introduce, for $i=1, \ldots, N$ and the sake of compactness, the notations below

$$
\begin{aligned}
& \xi^{i \top}=\left(z^{i^{\top}}, \xi^{i-1}{ }^{\top}\right) \\
& B_{i}^{\delta^{\top}}\left(\xi^{i}, u\right)=\left(G^{\delta^{\top}}{ }_{i}\left(z^{i}, \ldots, z^{1}, \xi, u\right), B_{i-1}^{\delta^{\top}}\left(\xi^{n-1}\right)\right) \\
& a_{i}^{\top}\left(\xi^{i}\right)=\left(f_{i}^{\top}\left(z^{i}\right)+\varphi^{\top}\left(z^{i}, \ldots, z^{1}, \xi\right), a_{i-1}^{\top}\left(\xi^{i-1}\right)\right) \\
& b_{i}^{\top}\left(\xi^{i}\right)=\left(g_{i}^{\top}\left(z^{i}, \ldots, z^{1}, \xi\right), b_{i-1}^{\top}\left(\xi^{i-1}\right)\right)
\end{aligned}
$$

with $\xi^{0}=\xi, B_{0}^{\delta}\left(\xi^{0}, u\right)=B^{\delta}(\xi, u), a_{0}\left(\xi^{0}\right)=a(\xi)$ and $b_{0}\left(\xi^{0}\right)=b(\xi)$.

Step 0: The dynamics 26c)-(26d) is $u$-average passive (at the sampling instants) with output $Y_{0}^{\delta}(\xi, u)=\frac{1}{\delta} \mathrm{L}_{B^{\delta}}(\cdot, u) U(\xi+(u))$ and the feedback $u=u_{0}^{\delta}(\xi)$ the solution of the implicit algebraic equality

$$
u=-\frac{1}{u} \int_{0}^{u} Y_{0}^{\delta}\left(\xi^{+}(v), v\right) \mathrm{d} v
$$

ensures S-GAS and S-LES of the $\xi$-dynamics in closed-loop.

Step 1: Denoting $V_{0}^{\delta}(\xi)=U(\xi)$, then for any $t \in[k \delta,(k+1) \delta[$ the dynamics

$$
\begin{aligned}
\dot{z}^{1}(t) & =f_{1}\left(z^{1}(t)\right)+\varphi_{1}\left(z^{1}(t), \xi(t)\right)+g_{1}\left(z^{1}(t), \xi(t)\right) u_{k} \\
\dot{\xi}(t) & =a(\xi(t))+b(\xi(t)) u_{k}
\end{aligned}
$$

is S-GS and its sampled-data equivalent is $u$-average passive from $u_{0}^{\delta}\left(\xi_{k}\right)$ with storage function

$$
V_{1}^{\delta}\left(\xi^{1}\right)=V_{1}^{\delta}\left(z^{1}, \xi\right)=W_{1}\left(z^{1}\right)+\Psi_{1}^{\delta}\left(z^{1}, \xi\right)+V_{0}^{\delta}(\xi)
$$

with

$$
\Psi_{1}^{\delta}\left(z^{1}, \xi\right)=\sum_{\ell=0}^{\infty} \int_{\ell \delta}^{(\ell+1) \delta} \mathrm{L}_{\varphi^{1}+u_{0}^{\delta}\left(\xi_{\ell}\right) g^{1}} W_{1}\left(z^{1}(s), \xi(s)\right) \mathrm{d} s
$$

and output $Y_{1}^{\delta}\left(\xi^{1}, u\right)=\frac{1}{\delta} \mathrm{L}_{B_{1}^{\delta}(\cdot, u)} V_{1}^{\delta}\left(\xi^{1}\right)$. The feedback $u=u_{1}^{\delta}\left(\xi^{1}\right)$ solution of the implicit algebraic equality

$$
u=-\frac{1}{u-u_{0}^{\delta}\left(\xi^{0}\right)} \int_{u_{0}^{\delta}\left(\xi^{0}\right)}^{u} Y_{1}^{\delta}\left(\xi^{1+}(v), v\right) \mathrm{d} v
$$

ensures S-GAS and S-LES of the equilibrium of (27).

At this point, one adds the $z^{2}$-dynamics to 27 in closed-loop under $u=u_{1}^{\delta}\left(\xi^{1}\right)$ and returns to Step 0 . This is iteratively performed until recovering the $z^{N}$-dynamics. For $i \geq 2$, one iterates the following steps.

Step i-1: The dynamics

$$
\dot{\xi}^{i-1}(t)=a_{i-1}\left(\xi^{i-1}(t)\right)+b_{i-1}\left(\xi^{i-1}(t)\right) u_{k}
$$

is S-GAS and S-LES under feedback $u_{i-1}^{\delta}\left(\xi^{i-1}\right)$.

Step $i$ : The dynamics

$$
\begin{aligned}
\dot{z}^{i}(t) & =f^{i}\left(z^{i}(t)\right)+\varphi^{i}\left(z^{i}(t), \xi^{i-1}(t)\right)+g^{i}\left(z^{i}(t), \xi^{i-1}(t)\right) u_{k} \\
\dot{\xi}^{i-1}(t) & =a_{i-1}\left(\xi^{i-1}(t)\right)+b_{i-1}\left(\xi^{i-1}(t)\right) u_{k}
\end{aligned}
$$

in closed loop with $u_{i-1}^{\delta}\left(\xi^{i-1}\right)$ verifies Assumptions 4.1 to 4.3 and is S-GS. Furthermore, it is $u$ average passive around $u_{i-1}^{\delta}\left(\xi^{i}\right)$ with output $Y_{i}^{\delta}\left(\xi^{i}, u\right)=\frac{1}{\delta} \mathrm{L}_{B_{i}^{\delta}(\cdot, u)} V_{i}^{\delta}\left(\xi^{i}\right)$ and storage function

$$
V_{i}^{\delta}\left(\xi^{i}\right)=V_{i}^{\delta}\left(z^{i}, \xi^{i-1}\right)=W_{i}\left(z^{i}\right)+\Psi_{i}^{\delta}\left(z^{i}, \xi^{i-1}\right)+V_{i-1}^{\delta}\left(\xi^{i-1}\right)
$$

with

$$
\begin{aligned}
& \Psi_{i}^{\delta}\left(z^{i}, \xi^{i-1}\right)=\sum_{\ell=0}^{\infty} \int_{\ell \delta}^{(\ell+1) \delta} \mathrm{L}_{\tilde{\varphi}^{i}\left(\cdot, \xi^{i-1}(s)\right)} W_{i}\left(z^{i}(s)\right) \mathrm{d} s \\
& \tilde{\varphi}^{i}\left(z^{i}, \xi^{i-1}\right)=\varphi^{i}\left(z^{i}, \xi^{i-1}\right)+g^{i}\left(z^{i}, \xi^{i-1}\right) u_{i-1}^{\delta}\left(\xi_{\ell}^{i-1}\right) .
\end{aligned}
$$

The feedback $u=u_{i}^{\delta}\left(\xi^{i}\right)$ solution to

$$
u=-\frac{1}{u-u_{i-1}^{\delta}\left(\xi^{i-1}\right)} \int_{u_{i-1}^{\delta}\left(\xi^{i-1}\right)}^{u} Y_{i}^{\delta}\left(\xi^{i+}(v), v\right) \mathrm{d} v
$$


makes (29) S-GAS and S-LES in closed loop.

Theorem 6.1: Let the continuous-time dynamics (25) be stabilizable in first approximation and suppose that, for any $i=$ $1, \ldots, N$, 25a verifies Assumptions 4.1 and 4.2. Moreover, let 25b verify Assumption 4.3 and be ZSD with output $y_{0}=$ $L_{b} U(\xi)$. Then, the following holds:

1) the sampled-data equivalent model $\sqrt{26}$ is $u$-average passive from $u_{N-1}^{\delta}\left(\xi^{N-1}\right)$ with output

$$
Y_{N}^{\delta}\left(\xi^{N}, u\right)=\frac{1}{\delta} \mathrm{L}_{B_{N}^{\delta}(\cdot, u)} V_{N}^{\delta}\left(\xi^{N}\right)
$$

and storage function

$$
V_{N}^{\delta}\left(\xi^{N}\right)=U(\xi)+\sum_{i=1}^{N}\left(W_{i}\left(z^{i}\right)+\Psi_{i}^{\delta}\left(z^{i}, \ldots, z^{1}, \xi\right)\right) ;
$$

2) the control law $u=u_{N}^{\delta}\left(\xi^{N}\right)$ solution to the implicit equality

$$
u=-\frac{1}{u-u_{N-1}^{\delta}\left(\xi^{N-1}\right)} \int_{u_{N-1}^{\delta}\left(\xi^{N-1}\right)}^{u} Y_{N}^{\delta}\left(\xi^{N+}(v), v\right) \mathrm{d} v
$$

makes the equilibrium of 25) S-GAS and S-LES.

Remark 6.1: Whenever $z^{i} \equiv 0$, one gets $\left.u_{i}^{\delta}\left(\xi^{i}\right)\right|_{z^{i}=0}=u_{i-1}^{\delta}\left(\xi^{i-1}\right)$. This implies that, for $\ell=1, \ldots, N$ and $z^{\ell} \equiv 0$, then $\left.u_{N}^{\delta}\left(\xi^{N}\right)\right|_{z^{\ell}=0}=u_{0}^{\delta}(\xi)$.

Remark 6.2: When specifying this procedure to strict-feedforward structures, at each step, the design yields the definition of a control that makes a certain stable manifold controlled invariant. We refer to [22], [23] for further details as in that case it follows the lines of the discrete-time case.

\section{APPRoXimate SOLUtions AND CONSTRUCTIVE ASPECTS}

Some computational aspects regarding the three steps of the design procedure are discussed below.

\section{A. The u-average passivity based stabilizing controller $u_{0}^{\delta}(\xi)$}

By definition of $B^{\delta}(\xi, u)$ and Remark 5.2, the $u$-average of the output $Y_{0}^{\delta}(\xi, u)$ in 14 can be described as a series expansion in powers of $\delta$ around the continuous-time output $y_{0}=\mathrm{L}_{b} U(\xi)$;namely, one gets

$$
\begin{aligned}
Y_{0}^{\delta, \text { av }}(\xi, u)= & \mathrm{L}_{b} U(\xi)+\frac{\delta}{2}\left(\left(\mathrm{~L}_{a}+u \mathrm{~L}_{b}\right) \mathrm{L}_{b} U(\xi)\right. \\
& \left.+\mathrm{L}_{b} \mathrm{~L}_{a} U(\xi)\right)+O\left(\delta^{2}\right) .
\end{aligned}
$$

By invoking the Implicit Function Theorem, a unique solution $u_{0}^{\delta}(\xi)$ to 15 exists in the form of the following series expansion in powers of $\delta$

$$
u_{0}^{\delta}(\xi)=u_{0}^{0}(\xi)+\sum_{i \geq 1} \frac{\delta^{i}}{(i+1) !} u_{0}^{i}(\xi) .
$$

Moreover, $u_{0}^{\delta}(\xi)$ can be computed as an inverse series around the continuous-time feedback solution (i.e., $u_{0}^{0}\left(\xi_{k}\right)=u_{0}\left(\xi_{k}\right)$ ). Exploiting the dependency in $\delta$ of the exact solution, an executive algorithm can be worked out to compute the successive additional terms $u_{0}^{i}(\xi)$ (see [24] for details). For the first terms, one gets

$$
\begin{aligned}
u_{0}^{0}\left(\xi_{k}\right)= & -\mathrm{L}_{b} U\left(\xi_{k}\right)=u_{0}\left(\xi_{k}\right) \\
u_{0}^{1}\left(\xi_{k}\right)= & -\mathrm{L}_{a+u_{0}^{0} b} \mathrm{~L}_{b} U\left(\xi_{k}\right)-\mathrm{L}_{b} \mathrm{~L}_{a} U\left(\xi_{k}\right) \\
= & \mathrm{L}_{a+u_{0}^{0} b} u_{0}\left(\xi_{k}\right)-\mathrm{L}_{b} \mathrm{~L}_{a} U\left(\xi_{k}\right) \\
u_{0}^{2}\left(\xi_{k}\right)= & \mathrm{L}_{a+u_{0}^{0} b}^{2} u_{0}\left(\xi_{k}\right)+\frac{3}{2} u_{0}^{1} \mathrm{~L}_{b} u_{0}\left(\xi_{k}\right) \\
& +\left(\mathrm{L}_{a+u_{0}^{0} b} \mathrm{~L}_{b}+\mathrm{L}_{b} \mathrm{~L}_{a}\right) \mathrm{L}_{a} U\left(\xi_{k}\right) .
\end{aligned}
$$

Whenever no confusion arises, for $i \geq 0, u_{0}^{i}$ will be denoting the constant value $u_{0}^{i}\left(\xi_{k}\right)$ for $t \in[k \delta,(k+1) \delta[$ (so implying $\dot{u}_{0}^{i}\left(\xi_{k}\right) \equiv 0$ and all the higher order time derivatives). 


\section{B. Construction of the sampled-data cross term $\Psi^{\delta}(z, \xi)$}

Some insights about the construction of the sampled-data pair $\left(\Psi^{\delta}, u_{0}^{\delta}\right)$ in terms of the continuous-time one $\left(\Psi, u_{0}\right)$ are given below.

Proposition 7.1: Let 8 verify Assumptions 4.1 to 4.3 , then 19 admits a solution $\Psi^{\delta}: \mathbb{R}^{n_{z}} \times \mathbb{R}^{n_{\xi}} \rightarrow \mathbb{R}$ of the form 18 around the continuous-time one defined in 99 , with

$$
\sum_{i \geq 1} \frac{\delta^{i}}{(i+1) !} \Psi_{i}(z, \xi)=\sum_{\ell=0}^{\infty} \int_{\ell \delta}^{(\ell+1) \delta}\left(u_{0}(\xi(s))-u_{0}^{\delta}\left(\xi_{\ell}\right)\right) \mathrm{L}_{\bar{g}}(\Psi+W)(s) \mathrm{d} s
$$

where $u_{0}^{\delta}\left(\xi_{\ell}\right)$ is described in 15 and $(z, \xi)=\left(z_{0}, \xi_{0}\right)$.

Proof: At first, we rewrite the continuous-time equality $\sqrt{11})$ as the equality between functions of the state $(z, \xi)$; i.e.

$$
\mathrm{L}_{\bar{f}} \Psi(z, \xi)+\mathrm{L}_{\varphi} W(z, \xi)=-u_{0}(\xi) \mathrm{L}_{\bar{g}}(\Psi+W)(z, \xi) .
$$

Thus, for any $u_{0}^{\delta}\left(\xi_{k}\right), k \geq 0$ one obtains the equality

$$
\mathrm{L}_{\bar{f}+u_{0}^{\delta}\left(\xi_{k}\right) \bar{g}} \Psi(z, \xi)+\mathrm{L}_{\varphi+u_{0}^{\delta}\left(\xi_{k}\right) g} W(z, \xi)=-\left(u_{0}(\xi)-u_{0}^{\delta}\left(\xi_{k}\right)\right) \mathrm{L}_{\bar{g}}(\Psi+W)(z, \xi)
$$

which proves that

$$
\mathrm{L}_{\bar{f}+u_{0}^{0}\left(\xi_{k}\right) \bar{g}} \Psi\left(z_{k}, \xi_{k}\right)+\mathrm{L}_{\varphi+u_{0}^{0}\left(\xi_{k}\right) g} W\left(z_{k}, \xi_{k}\right)=0
$$

in $O(\delta)$. This first argument shows that the solution $\Psi^{\delta}(\cdot)$ to $\sqrt{19}$ can be described through its series expansion around the continuous-time solution $\Psi(\cdot)$ as in $(18)$. On the other hand, by integrating 32$]$ over $[k \delta,(k+1) \delta[$ along the sampled-data dynamics $(16)$, one gets for any $k \geq 0$

$$
\Delta_{k} \Psi(z, \xi)-\Delta_{k} \Psi^{\delta}(z, \xi)=-\int_{k \delta}^{(k+1) \delta}\left(u_{0}(\xi(s))-u_{0}^{\delta}\left(\xi_{k}\right)\right) \mathrm{L}_{\bar{g}}(\Psi+W)(z(s), \xi(s)) \mathrm{d} s
$$

which gives, by definition of $\Psi(\cdot)$ and $\Psi^{\delta}(\cdot)$ in $97-20$

$$
\Psi^{\delta}(z, \xi)-\Psi(z, \xi)=-\sum_{\ell=0}^{\infty} \int_{\ell \delta}^{(\ell+1) \delta}\left(u_{0}(\xi(s))-u_{0}^{\delta}\left(\xi_{\ell}\right)\right) \mathrm{L}_{\bar{g}}(\Psi+W)(z(s), \xi(s)) \mathrm{d} s
$$

and thus 31 . $\triangleleft$

Equality 31 clearly emphasizes the impact of the piecewise constant nature of the feedback $u_{k}=u_{0}^{\delta}\left(\xi_{k}\right)$ over the redefinition of the cross term for the sampled-data dynamics with respect to the continuous-time couple $\left(u_{0}, \Psi\right)$. Expanding (31) and comparing the terms of the same power in $\delta$, one gets any $\Psi_{i}(z, \xi)$ is solution to a partial differential equation; for the first ones, one gets

$$
\begin{aligned}
\mathrm{L}_{\bar{f}_{0}(k)} \Psi_{1}= & -\left(u_{0}^{1}\left(\xi_{k}\right)-\left.\mathrm{L}_{\bar{f}_{0}(k)} u_{0}\right|_{k \delta}\right) \mathrm{L}_{\bar{g}}(W+\Psi) \\
\mathrm{L}_{\bar{f}_{0}(k)} \Psi_{2}= & -\left(u_{0}^{2}\left(\xi_{k}\right)-\left.\frac{3}{2} u_{0}^{1}\left(\xi_{k}\right) \mathrm{L}_{\bar{g}} u_{0}\right|_{k \delta}\right. \\
& \left.-\left.\mathrm{L}_{\bar{f}_{0}(k)}^{2} u_{0}\right|_{k \delta}\right) \mathrm{L}_{\bar{g}}(W+\Psi) \\
& -\left(\frac{3}{2} u_{0}^{1}\left(\xi_{k}\right)-\left.\mathrm{L}_{\bar{f}_{0}(k)} u_{0}\right|_{k \delta}\right) \mathrm{L}_{\bar{f}_{0}(k)} \mathrm{L}_{\bar{g}}(W+\Psi) \\
& -\frac{3}{2} u_{0}^{1}\left(\xi_{k}\right) \mathrm{L}_{\bar{g}} \Psi_{1}-\frac{3}{2} \mathrm{~L}_{\bar{f}_{0}(k)}^{2} \Psi_{1}
\end{aligned}
$$

where, for the sake of compactness, $\bar{f}_{0}(k)=\bar{f}+u_{0}^{0}\left(\xi_{k}\right) \bar{g}$ denotes the closed-loop dynamics under the piecewise constant feedback $u_{0}^{0}\left(\xi_{k}\right)$.

\section{The complete sampled-data stabilizing controller $u_{1}^{\delta}(z, \xi)$}

As already commented, a unique solution to the nonlinear equality (24) exists by direct application of the Implicit Function Theorem. The solution is provided as a formal series in powers of $\delta$. Exact solutions can be hardly computed so that only approximation of 34 can be implemented in practice. By rewriting $V^{\delta}(z, \xi)=V(z, \xi)+\sum_{i \geq 1} \frac{\delta^{i}}{(i+1) !} \Psi_{i}(z, \xi)$, one gets for the first terms

$$
u_{1}^{\delta}(z, \xi)=u_{1}^{0}(z, \xi)+\sum_{i \geq 1} \frac{\delta^{i}}{(i+1) !} u_{1}^{i}(z, \xi)
$$


with (when discarding the state dependency)

$$
\begin{aligned}
u_{1}^{0}= & -\mathrm{L}_{\bar{g}} V \\
u_{1}^{1}= & -\mathrm{L}_{\bar{f}+u_{1}^{0} \bar{g}} \mathrm{~L}_{\bar{g}} V-\mathrm{L}_{\bar{g}} \Psi_{1}-\mathrm{L}_{\bar{g}} \mathrm{~L}_{\bar{f}} V-u_{0}^{0} \mathrm{~L}_{\bar{g}}^{2} V \\
= & -\mathrm{L}_{\bar{g}} \Psi_{1}+\mathrm{L}_{\bar{f}+u_{1}^{0} \bar{g}} u_{1}^{0}-\mathrm{L}_{\bar{g}} \mathrm{~L}_{\bar{f}+u_{0}^{0} \bar{g}} V \\
u_{1}^{2}=- & \mathrm{L}_{\bar{g}} \Psi_{2}+\mathrm{L}_{\bar{f}+u_{1}^{0} \bar{g}}^{2} u^{0}-\frac{1}{2}\left(u_{1}^{1}+3 u_{0}^{1}\right) \mathrm{L}_{\bar{g}}^{2} V-\frac{3}{2} \mathrm{~L}_{\bar{f}+u_{0}^{0} \bar{g}} \mathrm{~L}_{\bar{g}} \Psi_{1} \\
& -\frac{3}{2} \mathrm{~L}_{\bar{g}} \mathrm{~L}_{\bar{f}+u_{1}^{0} \bar{g}} \Psi_{1}-\mathrm{L}_{\bar{f}+u_{0}^{0} \bar{g}} \mathrm{~L}_{\bar{g}} \mathrm{~L}_{\bar{f}+u_{1}^{0} \bar{g}} V-\mathrm{L}_{\bar{g}} \mathrm{~L}_{\bar{f}+u_{0}^{0} \bar{g}}^{2} V \\
& \quad-\left(u_{1}^{0}-u_{0}^{0}\right) \mathrm{L}_{\bar{g}}\left(\mathrm{~L}_{\bar{g}} \mathrm{~L}_{\bar{f}+u_{1}^{0} \bar{g}}+\mathrm{L}_{\bar{f}+u_{0}^{0} \bar{g}} \mathrm{~L}_{\bar{g}}\right) V .
\end{aligned}
$$

Remark 7.1: These approximate solutions coincide, under suitable modifications of the indices, to any other issued from any step of the general procedure sketched in Section VI

The stabilizing properties of approximate solutions of this form have been discussed in [29] and [30]. Specifically, $p^{\text {th }}$-order approximate feedback are defined as truncations at any finite order $p \in \mathbb{N}$ of the series expansion (34); namely,

$$
u_{1}^{\delta[p]}(z, \xi)=u_{1}^{0}(z, \xi)+\sum_{i=1}^{p} \frac{\delta^{i}}{(i+1) !} u_{1}^{i}(z, \xi) .
$$

Moreover, we refer to any $u_{1}^{i}(z, \xi)$ in 36 for $i \geq 1$ as corrector terms. Summarizing, it was proven that those feedbacks ensure practical asymptotic stability in closed-loop so that trajectories will converge onto a neighborhood of the origin whose size is determined by the length of $\delta^{p}$. Thus, the order of approximation needs to be chosen as a trade-off among computational effort and required performances in closed loop.

\section{Example}

Consider the simple cascade dynamics

$$
\dot{z}=\xi z ; \quad \dot{\xi}=u
$$

According to Section III-A, 37) admits an exactly computable sampled-data equivalent model

$$
z_{k+1}=e^{\delta\left(\xi_{k}+\frac{\delta}{2} u_{k}\right)} z_{k} ; \quad \xi_{k+1}=\xi_{k}+\delta u_{k}
$$

which clearly preserves the feedforward structure with nonlinear dependency in $u_{k}$. The equivalent $\left(F_{0}, G\right)$ representation takes the form

$$
\begin{aligned}
z^{+} & =e^{\delta \xi} z ; & & \frac{\mathrm{d} z^{+}(u)}{\mathrm{d} u}=\frac{\delta^{2}}{2} e^{-\delta\left(\xi^{+}(u)-\frac{\delta}{2} u\right)} z^{+}(u) \\
\xi^{+}=\xi ; & \frac{\mathrm{d} \xi^{+}(u)}{\mathrm{d} u}=\delta . & \text { r } & =\delta
\end{aligned}
$$

Finally, one verifies that the continuous-time dynamics 37 satisfies Assumptions 4.1 and 4.2 with $W(z)=\frac{1}{2} z^{2}$ and Assumption 4.3 with $y_{0}=\xi, U(\xi)=\frac{1}{2} \xi^{2}$.

On the basis of Section V-A one computes the average output $Y_{0}^{\delta}$,av $\left(\xi_{k}, u_{k}\right)=\xi_{k}+\frac{\delta}{2} u_{k}$ and the control solution $u_{0}^{\delta}=-\frac{2 \xi_{k}}{2+\delta}$ that is always well defined since $\delta \geq 0$ and recovers the continuous-time solution when $\delta=0$. The increment of the Lyapunov function along the $\xi$-trajectories gives $U\left(\xi_{k+1}\right)-U\left(\xi_{k}\right)=-\frac{4 \delta \xi_{k}^{2}}{(\delta+2)^{2}}$. It can be immediately verified that the right-hand side of (19) specifies as

$$
\begin{aligned}
\int_{0}^{\delta} z^{2}(t) \xi(t) \mathrm{d} t & =\int_{0}^{\delta}\left(1-\frac{2 t}{\delta+2}\right) e^{2 t\left(1-\frac{2 t}{\delta+2}\right) \xi_{k}} \mathrm{~d} t z_{k}^{2} \xi_{k}^{2} \\
& =\left(e^{2 \delta\left(1-\frac{\delta}{\delta+2}\right) \xi_{k}}-1\right) z_{k}^{2}
\end{aligned}
$$

Exploiting that

$$
z_{k}=e^{\left(1-\left(-\frac{\delta-2}{\delta+2}\right)^{k}\right) \xi} z ; \quad \xi_{k}=\left(-\frac{\delta-2}{\delta+2}\right)^{k} \xi
$$

20 reduces to

$$
\Psi^{\delta}(z, \xi)=\frac{1}{2}\left(e^{2 \xi}-1\right) z^{2}
$$


The final Lyapunov function $V(z, \xi)=\frac{1}{2} \xi^{2}+\frac{1}{2} e^{2 \xi} z^{2}$ verifies

$$
\Delta_{k} V(z, \xi) \leq U\left(\xi_{k+1}\right)-U\left(\xi_{k}\right)=-\frac{4 \delta}{(\delta+2)^{2}} \xi_{k}^{2} .
$$

Note that, in this case, the cross term $\Psi^{\delta}(z, \xi)$ in 39 ) verifies $\Psi_{1} \equiv \Psi_{2} \equiv 0$. Although it might seem restricting, approximations of the $\delta$-dependent cross term are unavoidable in order to compute and implement control solutions.

Finally, the overall control is computed by solving $\sqrt{24}$ in $O\left(\delta^{2}\right)$ as detailed in the previous sections. The output and the control approximated at the first order get the form

$$
\begin{aligned}
& Y^{\delta}(z, \xi)=\left(1+\frac{\delta}{2}\right) e^{2 \xi} z^{2}+\xi+O\left(\delta^{2}\right) \\
& u_{1}^{\delta}(z, \xi)=-e^{2 \xi} z^{2}-\xi+\frac{\delta}{2}\left(e^{4 \xi} z^{4}-2 \xi e^{2 \xi} z^{2}+\xi\right)+O\left(\delta^{2}\right) .
\end{aligned}
$$

From the above expressions it is easy to verify that for $\delta \rightarrow 0$ the continuous-time solution is recovered.

Simulations: The proposed control strategy is compared through simulations to the continuous-time one and the so-called emulated control (i.e., when implementing the continuous feedback by means of sample-and-hold devices). We implement approximate solutions of sampled-data feedback as in 36 for $p=1$ and $p=2$. The results are depicted in Figures 1(a), 1(b) and $1(\mathrm{c})$ for initial condition $\left(z_{0}, \xi_{0}\right)=(0.5,0.5)$. They clearly show that, as the sampling period increases, the proposed control strategy achieves very good performances (with smooth trajectories and even with only one corrector term) especially when the emulated one degrades or even fails (Figures $1(\mathrm{c})$ ). This empirically proves the efficiency of the sampled-data direct design (even with only one corrector term) when compared to mere sample-and-hold implementation [32], [33] of the continuous-time feedback. Moreover, contrarily to the emulated feedback, the evolutions of the Lyapunov function along the trajectories under the proposed sampled-data feedback are decreasing even when $\delta$ significantly increases. More in detail, the continuous-time $V(z, \xi)$ is no longer a Lyapunov function for the closed-loop system under emulated feedback. Finally, the simulated results underline the nested nature of the feedback in the sense of Remark 5.6, namely, first one drives $z$ to zero so recovering the integrator $\xi$-dynamics $\xi_{k+1}=\xi_{k}+\delta u_{k}$ evolving according to the feedback computed at the first step of the design. In this sense, when the emulated feedback is implemented (i.e., $u_{0}\left(\xi_{k}\right)=-\xi_{k}$ ) and $z \equiv 0$ the reduced linear $\xi$-dynamics is clearly unstable for higher values of $\delta$ as Figure 1(c) clearly underlines; in this same scenario, the dynamics under the proposed feedback $u_{1}^{\delta}(z, \xi)$ still shows good stabilizing performances.

\section{CONCLUSIONS}

This paper presents an iterative procedure for stabilizing general feedforward dynamics under sampling exploiting the preserved triangular structure. By suitably shaping the mappings and functions involved in the design, one shows how to construct a sampled-data stabilizing feedback under the same assumptions as in continuous time. The notion of $u$-average passivity around a nominal feedback is here introduced and is crucial for making the proposed design iterative. This study extends and concludes some previous works concerned with strict-feedforward systems [20] or more general classes under some stronger assumptions [21], [35]. The proposed methodology is in between the continuous and purely discrete-time cases as it requires less demanding assumptions for ensuring the existence of a stabilizing feedback by exploiting the properties of the continuous-time dynamics. The design is based on the definition of a cross term for the construction of a suitable control Lyapunov function and thus requires the explicit computation of the trajectories of the system over any sampling interval (as even in continuous time). Current work is toward the definition of modified Lyapunov functions to weaken this demand [42].

\section{REFERENCES}

[1] P. V. Kokolović, "The joy of feedback: nonlinear and adaptive," 1992.

[2] R. Sepulchre, M. Janković, and P. Kokotović, Constructive nonlinear control. Springer New York, 1997.

[3] P. Kokotović and M. Arcak, "Constructive nonlinear control: a historical perspective," Automatica, vol. 37, no. 5, pp. 637-662, 2001.

[4] A. Isidori, Nonlinear Control Systems. Springer-Verlag, 1995.

[5] F. Mazenc and L. Praly, "Adding an integration and global asymptotic stabilization of feedforward systems," IEEE Transactions on Automatic Control, vol. 41, no. 11, pp. 1559-1578, 1996.

[6] R. Sepulchre, M. Jankovic, and P. V. Kokotovic, "Integrator forwarding: a new recursive nonlinear robust design,” Automatica, vol. 33, no. 5, pp. 979-984, 1997.

[7] E. Panteley and A. Loria, “On global uniform asymptotic stability of nonlinear time-varying systems in cascade," Systems \& Control Letters, vol. 33, no. 2, pp. 131-138, 1998.

[8] M. Krstic, "Feedback linearizability and explicit integrator forwarding controllers for classes of feedforward systems," IEEE Transactions on Automatic Control, vol. 49, no. 10, pp. 1668-1682, Oct 2004.

[9] A. Astolfi and G. Kaliora, "A geometric characterization of feedforward forms," IEEE Transactions on Automatic Control, vol. 50, no. 7, pp. 1016-1021, 2005.

[10] F. Mazenc, "Stabilization of feedforward systems approximated by a non-linear chain of integrators," Systems \& Control Letters, vol. 32, no. 4, pp. 223-229, 1997.

[11] A. Astolfi and R. Ortega, "Immersion and invariance: a new tool for stabilization and adaptive control of nonlinear systems," IEEE Transactions on Automatic Control, vol. 48, no. 4, pp. 590-606, 2003. 

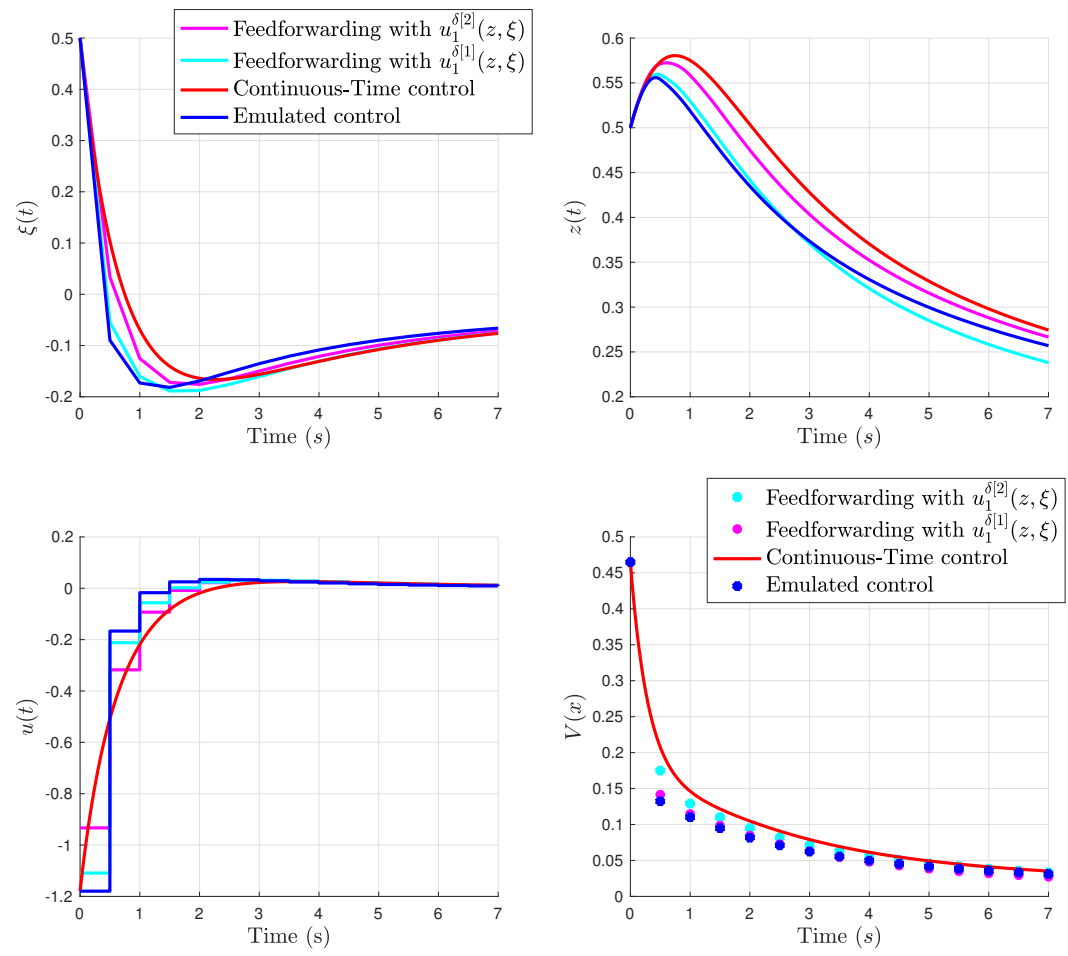

(a) $\delta=0.5 \mathrm{~s}$
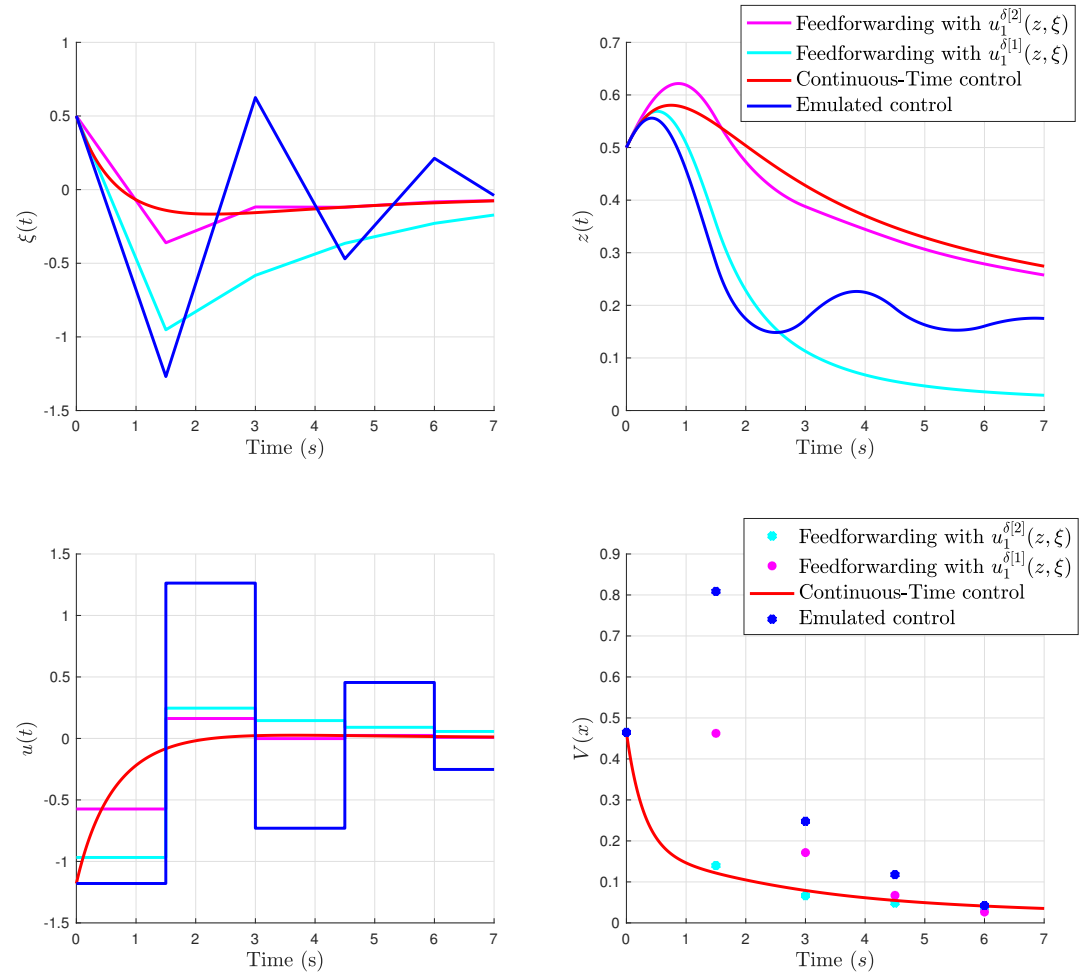

(b) $\delta=1.5 \mathrm{~s}$ 

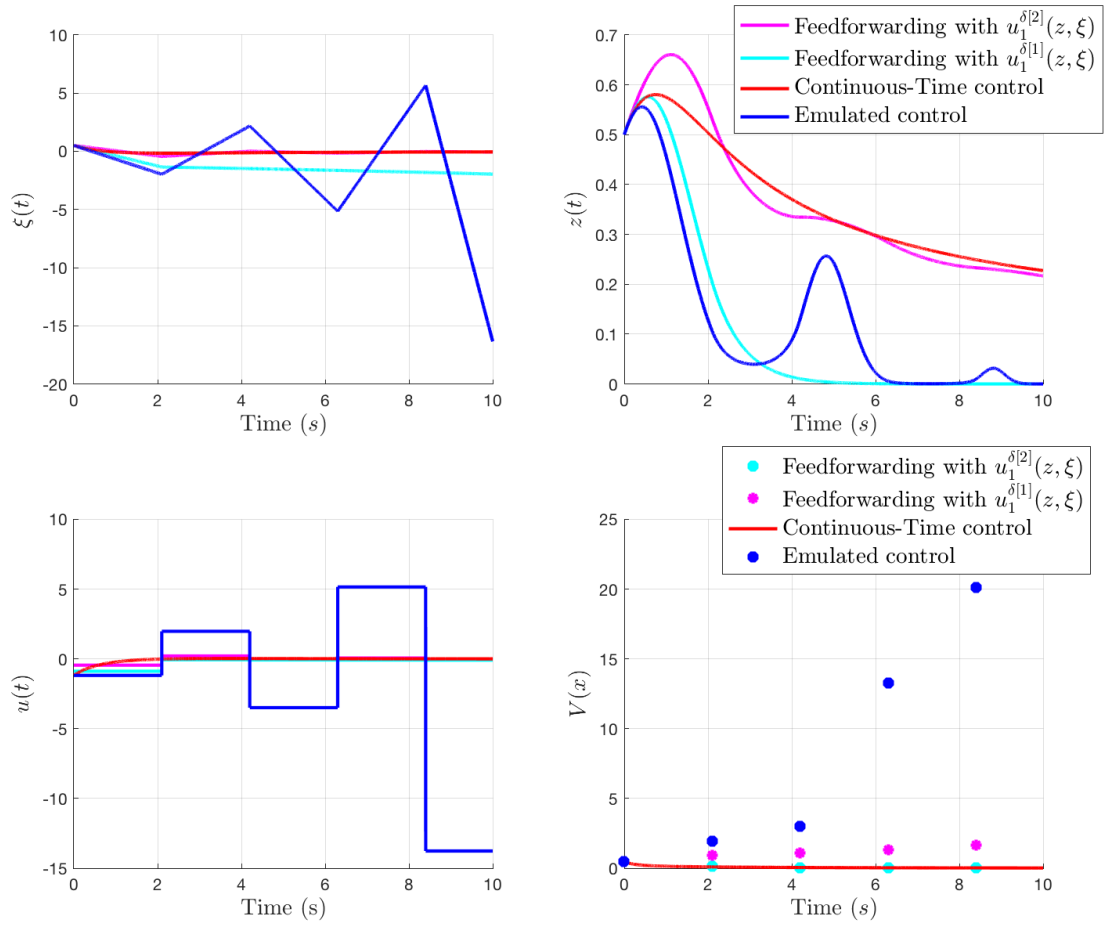

(c) $\delta=2.1 \mathrm{~s}$

[12] W. Lin and X. Li, "Synthesis of upper-triangular non-linear systems with marginally unstable free dynamics using state-dependent saturation," International Journal of Control, vol. 72, no. 12, pp. 1078-1086, 1999.

[13] W. Zha, S. Yan, C. Qian, and J. Zhai, "Global stabilization of a class of high-order upper-triangular nonlinear systems using dynamic state-feedback," IFAC-PapersOnLine, vol. 49, no. 18, pp. 730-735, 2016.

[14] J. Tsinias and M. Tzamtzi, "An explicit formula of bounded feedback stabilizers for feedforward systems," Systems \& Control Letters, vol. 43, no. 4, pp. 247-261, 2001.

[15] C. Qian and J. Li, "Global output feedback stabilization of upper-triangular nonlinear systems using a homogeneous domination approach," International Journal of Robust and Nonlinear Control, vol. 16, no. 9, pp. 441-463, 2006.

[16] X. Jia, W. Chen, and Z. Liu, "Semi-global output feedback stabilization of upper-triangular systems with uncertain output function," in Chinese Control and Decision Conference (CCDC). IEEE, 2016, pp. 6479-6484.

[17] F. Mazenc, A. Zemouche, and S.-I. Niculescu, "Observer with small gains in the presence of a long delay in the measurements," in 56th IEEE Conference on Decision and Control, CDC 2017, 2017.

[18] F. Mazenc and H. Nijmeijer, "Forwarding in discrete-time nonlinear systems," International Journal of Control, vol. 71, no. 5, pp. 823-835, 1998.

[19] T. Ahmed-Ali, F. Mazenc, and F. Lamnabhi-Lagarrigue, "Disturbance attenuation for discrete-time feedforward nonlinear systems," in Stability and Stabilization of Nonlinear Systems. Springer, 1999, pp. 1-17.

[20] S. Monaco and D. Normand-Cyrot, "Stabilization of nonlinear discrete-time dynamics in strict-feedforward form," in ECC 13, 2013 , pp. $2186-2191$.

[21] S. Monaco, D. Normand-Cyrot, and M. Mattioni, "Stabilization of feedforward discrete-time dynamics through immersion and invariance," in 2016 American Control Conference (ACC), July 2016, pp. 264-269.

[22] M. Mattioni, S. Monaco, and D. Normand-Cyrot, "Lyapunov stabilization of discrete-time feedforward dynamics," in 56th IEEE Conference on Decision and Control $(C D C)$, to appear, December 2017.

[23] —_ "Forwarding stabilization in discrete time," May 2017, submitted to Automatica.

[24] S. Monaco and D. Normand-Cyrot, "Advanced tools for nonlinear sampled-data systems, analysis and control," European Journal of Control, vol. 13, no. $2-3$, pp. $221-241,2007$.

[25] I. Karafyllis and C. Kravaris, "Global stability results for systems under sampled-data control," International Journal of Robust and Nonlinear Control, vol. 19, no. 10, pp. 1105-1128, 2009. [Online]. Available: http://dx.doi.org/10.1002/rnc.1364

[26] L. Hetel, C. Fiter, H. Omran, A. Seuret, E. Fridman, J.-P. Richard, and S. I. Niculescu, "Recent developments on the stability of systems with aperiodic sampling: An overview," Automatica, vol. 76, pp. 309-335, 2017.

[27] H. Omran, L. Hetel, J.-P. Richard, and F. Lamnabhi-Lagarrigue, "Stability analysis of bilinear systems under aperiodic sampled-data control," Automatica, vol. 50, no. 4, pp. 1288-1295, 2014.

[28] H. Omran, L. Hetel, M. Petreczky, J.-P. Richard, and F. Lamnabhi-Lagarrigue, "Stability analysis of some classes of input-affine nonlinear systems with aperiodic sampled-data control," Automatica, vol. 70, pp. 266-274, 2016.

[29] V. Tanasa, S. Monaco, and D. Normand-Cyrot, "Backstepping control under multi-rate sampling," IEEE Transactions on Automatic Control, vol. 61, no. 5, pp. 1208-1222, May 2016.

[30] M. Mattioni, S. Monaco, and D. Normand-Cyrot, "Immersion and invariance stabilization of strict-feedback dynamics under sampling," Automatica, vol. 76, pp. $78-86,2017$

[31] W. Lin, W. Wei, and G. Ye, "Global stabilization of a class of nonminimum-phase nonlinear systems by sampled-data output feedback," IEEE Transactions on Automatic Control, vol. 61, no. 10, pp. 3076-3082, 2016.

[32] H. Du, C. Qian, and S. Li, "Global stabilization of a class of uncertain upper-triangular systems under sampled-data control," International Journal of Robust and Nonlinear Control, vol. 23, no. 6, pp. 620-637, 2013. 
[33] D. Zhang and Y. Shen, "Global output feedback sampled-data stabilization for upper-triangular nonlinear systems with improved maximum allowable transmission delay," International Journal of Robust and Nonlinear Control, vol. 27, no. 2, pp. 212-235, 2017.

[34] S. Monaco and D. Normand-Cyrot, "Nonlinear average passivity and stabilizing controllers in discrete-time," Systems \& Control Letters, vol. 60, pp. 431-439, 2011

[35] M. Mattioni, S. Monaco, and D. Normand-Cyrot, "Sampled-data stabilization of feedforward dynamics with Lyapunov cross-term," in 2016 IEEE 55th Conference on Decision and Control (CDC), Dec 2016, pp. 1322-1327.

[36] S. Monaco and D. Normand-Cyrot, "On the sampling of a linear analytic control system," in 24th IEEE Conference on Decision and Control (CDC), vol. 24. IEEE, 1985, pp. 1457-1462.

[37] J. Yuz and G. C. Goodwin, Sampled-data models for linear and nonlinear systems. Springer-Verlag London, 2014.

[38] D. Nesic and A. R. Teel, "A framework for stabilization of nonlinear sampled-data systems based on their approximate discrete-time models," IEEE Transactions on Automatic Control, vol. 49, no. 7, pp. 1103-1122, 2004.

[39] S. Adly, B. Brogliato, and B. K. Le, "Implicit euler time-discretization of a class of lagrangian systems with set-valued robust controller," Journal of Convex Analysis, vol. 23, no. 1, pp. 23-52, 2016.

[40] S. Monaco, C. Normand-Cyrot, and C. Califano, "From chronological calculus to exponential representations of continuous and discrete-time dynamics: A Lie-algebraic approach," IEEE Transactions on Automatic Control, vol. 52, no. 12, pp. 2227 -2241, 2007.

[41] A. Pavlov and L. Marconi, "Incremental passivity and output regulation," Systems \& Control Letters, vol. 57, no. 5, pp. 400 - 409, 2008.

[42] M. Malisoff and F. Mazenc, Constructions of Strict Lyapunov Functions, ser. Communications and Control Engineering. Springer, 2009. 